\author{
Universidade de São Paulo \\ Instituto de Física
}

\title{
Investigação óptica e estrutural de lipoproteínas de baixa densidade de pacientes diabéticos com e sem periodontite
}

\section{Ghadeer Albattarni}

\section{Orientador:}

Prof. Dr. Antonio Martins Figueiredo Neto

Banca examinadora:

Profa. Dra. Maria Cristina Izar (UNIFESP - EPM)

Profa. Dra. Maria Aparecida Neves Jardini (UNESP - S. José dos Campos)

Dissertação de mestrado apresentada ao Instituto de Física para a obtenção do título de Mestre em Ciências

São Paulo 
FICHA CATALOGRÁFICA

Preparada pelo Serviço de Biblioteca e Informação do Instituto de Física da Universidade de São Paulo

Albattarni, Ghadeer

Investigação óptica e estrutural de lipoproteínas de baixa densidade de pacientes diabéticos com e sem periodontite. São Paulo, 2018.

Dissertação (Mestrado) - Universidade de São Paulo, Instituto de Física, Depto. de Física Experimental.

Orientador: Prof. Dr. Antonio Martins Figueiredo Neto

Área de Concentração: Grupo de Fluídos Complexos.

Unitermos: 1. Varredura-Z; 2. Lipoproteína; 3. LDL; 4. HDL;

5. Colesterol.

USP/IF/SBI-106/2018 


\section{University of São Paulo \\ Institute of Physics}

\section{Optical and structural investigation of low-density lipoprotein of diabetic patients with and without periodontitis}

\section{Ghadeer Albattarni}

Advisor: Prof. Dr. Antonio Martins Figueiredo Neto

Master thesis submitted to the Institute of Physics to obtain the title of Master of Science

São Paulo

2018 



\section{ACKNOWLEDGMENTS}

- Prof. Dr. Antonio Martins Figueiredo Neto for his supervising of this work. I am really grateful for his great support throughout this research.

- Group São José dos Campos (FOSJC-UNESP) for the cooperation in this work.

- All my colleagues: Andre, Ana, Wagner, Luiz, Edreson, Eduardo, for the assistance and useful discussion.

- All the officials in this group: Danial, Dennys, Fernando, Arlando, Juliana, Ellen for the assistance and useful discussion.

- All my family: my mother, brothers, sister, for their continuous love and great support to continue.

- My daughter who was always with me despite thousands of miles away. 


\section{Resumo}

Alta concentração de lipoproteína modificada de baixa densidade (LDL) é um dos fatores que aumentam o risco de doenças cardíacas. As concentrações mais elevadas de LDL modificada estão associadas à aterosclerose nas veias de sangue. A técnica de Z- scan (ZS) foi utilizada para investigar a resposta óptica não-linear da lipoproteína modificada de baixa densidade (moLDL). As características estruturais do $\mathrm{mLDL}$ foram estudadas por espalhamento dinâmico de luz. Nesta pesquisa, os experimentos foram realizados em amostras de LDL de 24 pacientes com diabetes e gengivite (grupo 1) e 24 pacientes com diabetes e periodontite

(grupo 2). Os experimentos foram repetidos após 6 meses sem qualquer tratamento para o grupo 1 e com um tratamento periodontal para o grupo 2 . Os resultados mostram que a amplitude da curva Z-scan no grupo 2 é menor que a do grupo 1. Após 6 meses de tratamento periodontal a amplitude é maior do que antes, depois a concentração de LDL modificado é reduzida nos pacientes tratados. Portanto, o risco cardiovascular para aterosclerose é diminuído após o tratamento periodontal. Além disso, os resultados do espalhamento dinâmico de luz mostram que não há nenhuma mudança estrutural significativa no moLDL entre os dois grupos antes e após 6 meses. 


\begin{abstract}
High-concentration of modified low-density lipoprotein (LDL) is one of the factors that increases the risk of heart disease. The higher concentrations of modified LDL are associated with the atherosclerosis in the bloodstream. The Z-scan (ZS) technique was used to investigate the nonlinear optical response of modified low-density lipoprotein (moLDL). The structural characteristics of moLDL were studied by dynamic light scattering. In this work, the experiments were carried out in LDL solution from 24 patients with diabetes and gingivitis (group 1) and 24 patients with diabetes and periodontitis (group 2). The experiments had been repeated after 6 months without any treatment for group 1 and with a periodontal treatment for group 2. The results show that the amplitude of Z-scan curve in group 2 is lower than that of group 1. After 6 months of periodontal treatment the amplitude is getting higher than before, then the concentration of modified LDL is reduced in the patients treated. Therefore, the cardiovascular risk for atherosclerosis is decreased after the periodontal treatment. Also, dynamic light scattering results show that there is not a significant structural change in moLDL between the two groups before and after 6 months.
\end{abstract}




\section{Nomenclature}

\section{List of symbols:}

\begin{tabular}{cl}
$\alpha$ & Light absorption coefficient. \\
$P$ & Beam power \\
$\kappa$ & Thermal conductivity of the sample \\
$r$ & Radial distance to the beam axis and exponential integral function. \\
$E i(x)$ & Exponential integral function. \\
$D_{t h}$ & Thermal diffusivity. \\
$\rho$ & Mass density. \\
$C_{p}$ & Specific heat capacity of the sample. \\
$t_{t h}$ & Characteristic time of heat diffusion. \\
$n_{0}$ & Linear refractive index. \\
$d n / d T$ & Thermo-optic coefficient. \\
$l e f f$ & Effective propagation length of the sample. \\
$L$ & Sample thickness. \\
$z_{0}$ & Rayleigh length \\
$\lambda$ & Light wavelength and \\
$w_{0}$ & the beam waist at the focal position $(\mathrm{z}=0)$. \\
$I_{0}$ & Initial intensity. \\
$I_{T_{r}}$ & Transmitted intensity. \\
$\alpha$ & Linear absorption coefficient. \\
$A$ & Absorbance \\
$t$ & Delay time. \\
$\Gamma$ & Decay constant. \\
$D$ & Diffusion coefficient. \\
$q$ & Modulus of scattering vector. \\
$\mathrm{r}$ & Hydrodynamic radius of the nanoparticle. \\
$\mathrm{K}_{\mathrm{B}}$ & Boltzmann's constant. \\
$\mathrm{T}$ & Sample's equilibrium temperature. \\
$\eta(\mathrm{t})$ & Viscosity of the liquid. \\
& \\
\hline &
\end{tabular}




\section{List of abbreviations:}

LDL

ZS

moLDL

CVD

WHO

QM

VLDL

HDL

UV

DLS

NNLS low-density lipoprotein.

Z-scan technique.

modified low-density lipoprotein.

cardiovascular diseases.

the World Health Organization.

Quilomicrons.

very low-density lipoprotein.

high-density lipoprotein.

Ultra-violet.

Dynamic light scattering.

Non-Negatively constrained Least Squares. 


\section{List of figures}

1.1 Image of low-density lipoprotein (LDL). 2

2-1 Schematic drawing of the Z-scan technique. 8

2-2 Illustration of the thermal-lens effect for a sample having a 9 negative $d n / d T$.

2-3 The Z-scan typical curve of the thermal-lens effect. Normalized Transmittance as a function of z-position for two samples with different signs of $d n / d T$.

2-4 Graph illustrating the meaning of $\Delta \mathrm{T}_{p-v}$.

$\begin{array}{ll}\text { 2-5 } & \text { Experimental setup of Z-scan technique . } \\ \end{array}$

2-6 Configuration of the Z-scan arrangement through the 16

2-7 Z-scan_chopper_new software with the data collected from the 17 oscilloscope .

2-8 Typical oscilloscope's records of the transmittance, as a function of time, of a sample with negative $d n / d T$.

2-9 Illustrative scheme of the transmission when the light passes 19 through a material.

2-10 Sketch of the DLS setup. 22

4-1 Comparison of Z-Scan typical curve between some patients. (a) 31 experimental data and theoretical fitting. (b) patients with diabetes and periodontitis versus patients with only diabetes. (c) evolution of the z-scan signal after six months.

4-2 Box plot of the $\theta$ values of all the patient from group 1 and group 2 in the beginning (time 0 ).

4-3 Box plot of the $\theta$ values of all patients from group 2 before and $\quad 34$ after 6 months of treatment.

4-4 Box plot of the $\theta$ values of all the patient from group 1 and group $\quad 35$ 2 after 6 months (time 6 ).

4-5 Box plot of the $\theta$ values of all patients from group 1 before and after 6 months.

4-6 The absorbance (A) as a function of wavelength for LDL samples from some patients.

4-7 Box plot of the $\alpha$ values of all the patient from group 1 and group 
2 in the beginning (time 0 ).

4-8 Box plot of the $\alpha$ values of all the patient from group 2 before and after 6 months of treatment.

4-9 Box plot of the $\alpha$ values of all patients from group 1 and group 2 after 6 months.

4-10 Box plot of the $\alpha$ values of all patients from group 1 before and after 6 months.

4-11 Temperature dependence of the refractive index of the LDL sample from one patient.

4-12 Box plot of the thermal conductivity $(k)$ values of all the patient from group 1 and group 2 in the beginning (time 0 ).

4-13 Box plot of the thermal conductivity $(k)$ values of all the patient from group 1 and group 2 after 6 months.

4-14 Box plot of the thermal conductivity $(k)$ values of all the patient from group 1 before and after 6 months.

4-15 Box plot of the thermal conductivity $(k)$ values of all the patient from group 2 before and after 6 months of treatment.

4-16 Measured (blue points) and fitted (black line) autocorrelation function using the NNLS method (a). Particle-size distribution histograms weighted by number (b), volume (c), and intensity of scattered light (d). Data and analysis done for patient 48 at time 0 .

4-17 (a) Box plot of the sizes of LDL particles values of the patient from group 1 and group 2 in the beginning (time 0 ). (b) Intensity autocorrelation functions measured by dynamic light scattering (DLS) for the samples of patients.

4-18 Box plot of the size of LDL particles values of the patient from group 1 and group 2 after 6 months.

4-19 Box plot of the size of LDL particles values of the patient from group 2 before and after 6 months of treatment.

4-20 Box plot of the size of LDL particles values of the patient from group 1 before and after 6 months. 


\section{Content}

Resumo III

Abstract V V

Nomenclature. $\quad$ VII

List of figures

1- Introduction

1-1 Characteristics of Low-Density Lipoprotein 1

1-2 Diabetes and Cardiovascular Disease 3

1-3 Diabetes and Periodontitis 4

\section{2- Experimental techniques}

2-1 Z-scan technique $\quad 7$

2-1-1 Z-scan

2-1-2 Thermal-lens model $\quad 10$

2-1- 3 Experimental setup 14

2-2- linear absorbance experiment 18

2-2-1 Measurement of the linear absorption coefficient 18

2-2-2 Experimental setup 20

2-3 Measurement of the thermo-optic coefficient 21

2-4 Dynamic light scattering technique 21

2-4-1 Experimental setup 24

\section{3-Samples}

3-1 Selection criteria 25

3-2 Separation of lipoproteins 27

3-3 Biochemistry analyses 28

3-4 Statistical analysis 28

4- Experimental results and discussion

4-1 Z-scan 30

4-2 linear absorption $\quad 36$

4-3 measurement of the thermo-optic coefficient 41

4-4 thermal conductivity $\quad 42$

4-5 Dynamic light scattering $\quad 45$

5- Conclusion $\quad 50$

References 


\section{1- Introduction}

\section{1-1 Characteristics of Low-Density Lipoprotein}

Nowadays, cardiovascular diseases (CVD) represent one of the main causes of morbidity and mortality in the world. According to the World Health Organization (WHO), the increase in the incidence of CVD tends to persist, worsening the health conditions of developing countries [1].

Dyslipidemia is one of the major risk factors for the CVD. From the pathophysiological point of view, dyslipidemia results from alteration in the metabolism of the lipoproteins. These particles are responsible of the transportation of lipids in plasma, and are mainly composed of lipids and proteins (called apolipoproteins). Lipoproteins are classified into 4 major classes, according to their density, in quilomicrons (QM), very low-density lipoprotein (VLDL), low-density lipoprotein (LDL) and high-density lipoprotein (HDL). In this context, dyslipidemia, especially is due to the elevation of LDL concentration in plasma, is considered a fundamental risk factor for CVD [2].

The main function of LDL is transporting cholesterol from the liver to the peripheral tissues of the body. The diameter of the LDL is between 19 and $25 \mathrm{~nm}$ [3]. The LDL contains approximately $50 \%$ cholesterol (free and esterified), $25 \%$ proteins, $20 \%$ phospholipids and 5\% triglycerides. Over 95\% of the LDL mass is the apoliprotein B100 (apo B-100, $549 \mathrm{kDa}$ ), each LDL particle contains only one molecule of apo B1000 [4]. The molecular mass of LDL is in the range from 2.4-3.9 MDa [5]. 
Lipoproteins have micellar-like structure: the surface is formed by a monolayer of phospholipids, free cholesterol and antioxidants (the main ones being alphatocopherol and beta-carotene); the nucleus is hydrophobic, formed by ester of cholesterol and triglycerides, in different proportions in each lipoprotein [2]. In LDL, between 40 and $60 \%$ of the surface area of the phospholipids monolayer is covered by apolipoprotein B-100 [6]. Apo B-100 is exposed at the surface allowing receptor recognition [7].

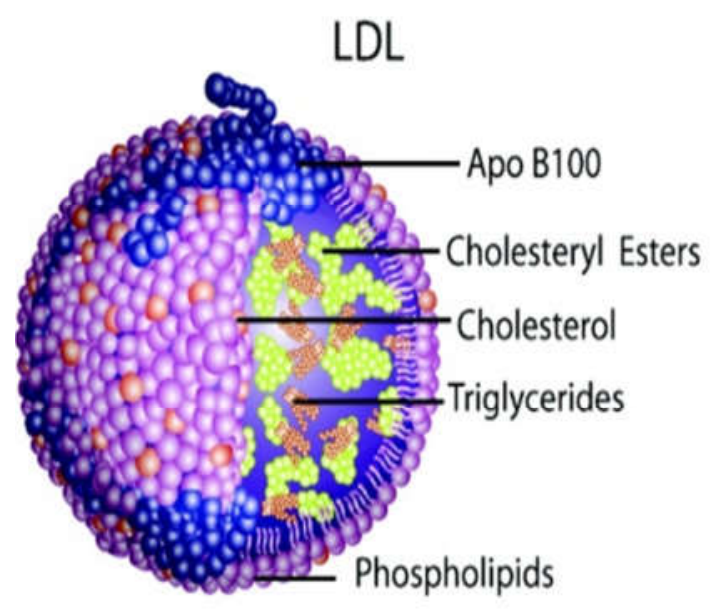

Figure 1.1: Image of low-density lipoprotein (LDL)

The LDL particle quantity is heterogeneous according to size, density and composition. Distinct subpopulations vary in isoelectric point, electrical charge, hydrodynamic properties and immunoreactivity [8].

The subfraction composition varies between individuals. The structure of LDL particles of different densities varies with respect both to the size of the core and the width of the surface shell [9]. 


\section{1-2 Diabetes and Cardiovascular Disease}

Diabetes is a serious, chronic disease that occurs either when the pancreas does not produce enough insulin (a hormone that regulates blood glucose), or when the body cannot effectively use the insulin it produces. Insulin is a hormone made by the pancreas, which acts like a key to let glucose from the food we eat pass from the blood stream into the cells in the body to produce energy. All carbohydrate foods are broken down in glucose in the blood. Insulin helps glucose to get into the cells to generate energy. Not being able to produce insulin or inability to use it effectively leads to raised glucose levels in the blood (known as hyperglycemia). Hyperglycemia, may, over time, leads to a serious damage to the heart, blood vessels, eyes, kidneys, nerves and odontological health.

More than 400 million people live with diabetes. Diabetes is one of the leading causes of mortality, affecting at least 425 million adults worldwide. Approximately, $79 \%$ of adults with diabetes are living in low- and middle-income countries, in additional, 352 million people are at risk of developing type 2 diabetes. The proportion of people with type 2 diabetes is increasing in most countries [10]. The prevalence of DM2 is projected to rise from the current estimates 240 million affected to some 380 million by 2025 [11].

There are two types of the diabetes: type 1 and type $2[12,13]$. Diabetes mellitus type 1 (DM1), previously known as insulin-dependent, juvenile or childhood-onset diabetes, is characterized by deficient insulin production in the body. People with DM1 require daily administration of insulin to regulate the amount of glucose in their blood. Diabetes mellitus type 2 (DM2), previously called non-insulin-dependent, 
results from the body's ineffective use of insulin. DM2 patients account for the vast majority of people with diabetes around the world $[12,13]$.

Adults with diabetes historically have two or three times higher rates of CVD than adults without diabetes [14]. The risk of cardiovascular disease increases continuously with rising fasting plasma glucose levels, even before reaching sufficient levels for diagnosis [15]. Protein glycation that occurs in diabetic patients is regarded as one of the key factors in the pathogenesis of diabetic complications, including accelerated atherosclerosis and CVD [16]. Although nonenzymatic glycosylation and oxidation are increased in DM2, these features would not be directly involved in the generation of modified LDL [17].

\section{1-3 Diabetes and Periodontitis}

Periodontitis is characterized by the destruction of connective tissue and dental bone support following an inflammatory host response secondary to infection by periodontal bacteria $[18,19]$. Overall, periodontal disease affects about $20-50 \%$ of the population around the globe [20].

High concentrations of cholesterol and the action of oral bacteria in the process of atherosclerosis or the participation of acute-phase proteins may increase in chronic periodontitis [21]. Also, periodontal disease is able to increase the risk of vascular diseases due to the fact of increasing the infection by subgingival microbial species.

Diabetes mellitus is one of the systemic risk factors for periodontal diseases, which can play a major role in initiation and progression of the disease [22]. DM2 and periodontitis present the same inflammatory etiopathogenesis [23]. This fact

demonstrate a bidirectional relationship, since DM2 affects the severity of 
periodontitis, and this may contribute to the individual's total inflammatory load, influencing the natural course of DM2 [24].

The association between diabetes mellitus and periodontite can be explained as a manifestation of systemic inflammation and the corresponding mechanisms of insulin sensitivity and glucose dynamics [25]. The increased severity or chronicity of periodontite increases the insulin resistance and aggravates glycemic control [26]. In a recent study with 200,026 patients with periodontite and 154,824 subjects with a healthy oral status, periodontite was significantly positively related to diabetes mellitus [27].

High cholesterol LDL favor atherosclerosis that is an inflammatory disease that develops in the large arteries, and it is responsible for cardiovascular events. The hyperglycemia and oxidative stress can be found in individuals with DM2 and chronic periodontitis. In parallel, the chronic periodontitis is an inflammatory disease and it could have a negative effect on glycaemic control in favor of a prediabetes stage and CVD. This profile can generate modified LDL by oxidation (high free-radicals concentration) and/or glycation (hyperglycemic situations), therefore, causes a high risk for the CVD [28-30]. So it was hypothesized that the LDL profile in patients with periodontitis and DM2 is different compared with patients with DM2.

Several studies showed that modified LDL (moLDL) has a role in the formation and development of atherosclerotic lesions in humans [31,32]. It has been demonstrated that the atherosclerosis is associated with higher concentrations of modified LDL in the bloodstream [33,34]. Complex Fluid Group of the USP Institute of Physics (GFCX-IFUSP) succeeded in determining the amount of moLDL in the bloodstream by introducing a technique that is known as the Z-scan technique [35-37]. The 
importance of these methods comes through their applicability on biological samples. Using Z-scan technique the studies showed that it can be differentiate between the native LDL and moLDL solutions, where the nonlinear optical responses of both of them are different [35]. Monteiro et all showed in their paper the relationship between periodontitis and an increased risk for CAD [30]. Also, they studied the behavior of moLDL solution from patients with periodontitis and showed that it can be decreased the cardiovascular risk for atherosclerosis after a periodontal treatment [38].

In this project, we study the behavior of moLDL solution and his nonlinear optical response in patients with diabetes and periodontits and compared with patients with diabetes and gingivitis. We study also, the improvement of moLDL solution in patients with diabetes and periodontits after 6 months periodontal treatment. Several parameters are studied as linear absorption coefficient, thermo-optical coefficient and thermal conductivity. The structural characteristics of moLDL is studied by a dynamic light scattering (DLS). 


\section{2- Experimental technique}

\section{2-1 Z-scan technique}

\section{2-1-1 Z-scan}

The first to develop and describe the Z-scan technique was Sheik-Bahae et al in $1989[35,36]$. The Z-scan technique is considered a simple and direct way to measure the nonlinear effects on the light transmittance through a material when it is illuminated by a Gaussian laser beam. This technique measures the nonlinear absorption, the sign and magnitude of the nonlinear refractive index $[35,36]$. Using this method it is possible to study the thermo-optical properties of various materials such as liquid crystals, colloidal solutions [39-43], semiconductor and nanomaterials [44-47]. It has recently been applied to biological solutions like low-density lipoprotein [38].

In general Z-scan technique is based on the principles of spatial-beam distortion. A tightly focused Gaussian laser beam passes the sample, which is moved through the focus of the beam, along the z-axis. The changing in the transmittance through the sample as a function of the sample position ( $\mathrm{z}$ ) is measured by a photodetector. This detector has a finite aperture and is placed in the far field. Figure 2-1 shows the basic setup of the Z-scan experiment. 


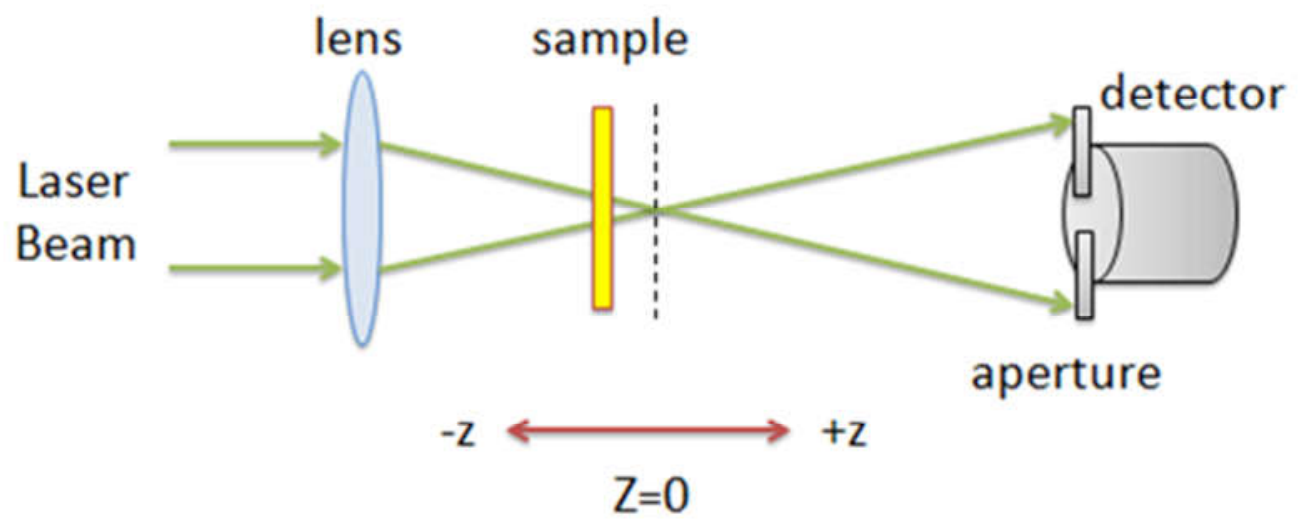

Fig 2-1: Schematic drawing of the Z-scan technique

When the beam reaches the sample, it absorbs the light and converts it into heat, generating a temperature gradient, where the maximum temperature is in the center of the beam (illuminated region) and decreases towards the edge. This behavior induces a refraction-index gradient inside the sample, which is the thermal lens effect that occurs on the time scale of milliseconds. The Gaussian beam also is pulsed by a chopper, providing square periodic pulses. We call the refractive index variation as a function of the temperature variation thermo-optic coefficient (dn/dT).

If the sample is a medium with $\mathrm{dn} / \mathrm{dT}>0$ then it will act as a converging lens. This effect is called self-focusing effect. If the medium of a sample has $\mathrm{dn} / \mathrm{dT}<0$ it will act as a diverging lens. This effect is called self-defocusing effect.

The sample with nonlinear refractive index will interact with the light as a lens. Let's consider a material with $\mathrm{dn} / \mathrm{dT}<0$ and a thickness smaller than Rayleigh length $\left(\mathrm{z}_{0}\right)$. When the sample is far from the focus $(\mathrm{z}<0)$ the beam intensity is low and the nonlinear refraction is negligible, therefore the transmittance remains the same. As the sample is moved towards the focus (on $-\mathrm{z}$ positions) (Figure 2-a), the beam intensity is increased causing a negative lensing effect (self-defocusing effect) and a decrease in the beam divergence. Thus, the transmittance measured at the aperture is increased (Figure 2-b). When the sample is at the focus $(z=0)$ no effect 
occurs on the beam (Figure 2-c). As the sample moves away from the focus (on $+\mathrm{z}$ positions) the self-defocusing effect occurs within the sample, leading to an increase in the beam divergence. Therefore, a decrease in the transmittance is measured at the aperture (Figure 2-d). Finally, when the sample moves far from the focus $(\mathrm{z}>0)$ the beam intensity is low and the nonlinear refraction also is negligible. Hence the transmittance returns to the initial linear value (Figure 2-e). As a result of negative $\mathrm{dn} / \mathrm{dT}$ nonlinearity a pre-focal transmittance maximum (peak), followed by a post-focal transmittance minimum (valley), is the Z-scan signature. In the other case, the Z-scan signature of positive $\mathrm{dn} / \mathrm{dT}$ nonlinearity corresponds to valley followed by a peak as shown in Figure 2-3.
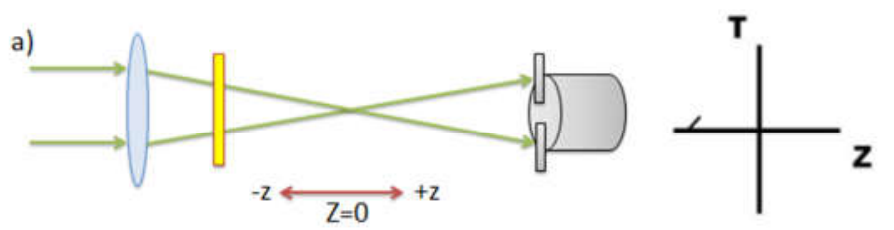

b)

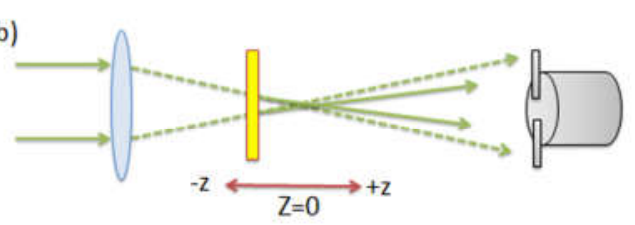

c)

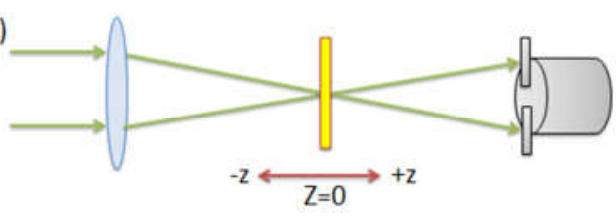

d)

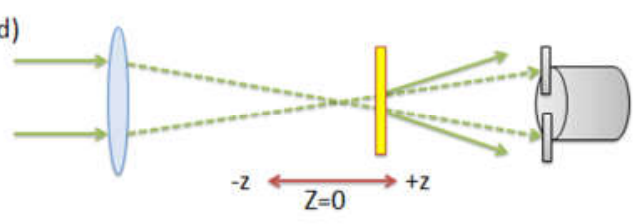

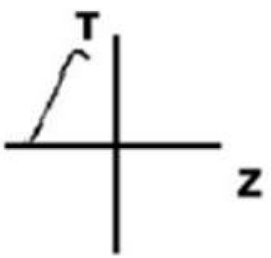
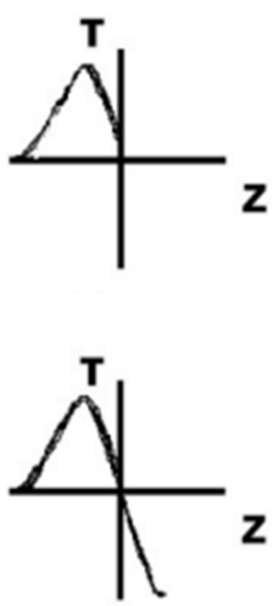

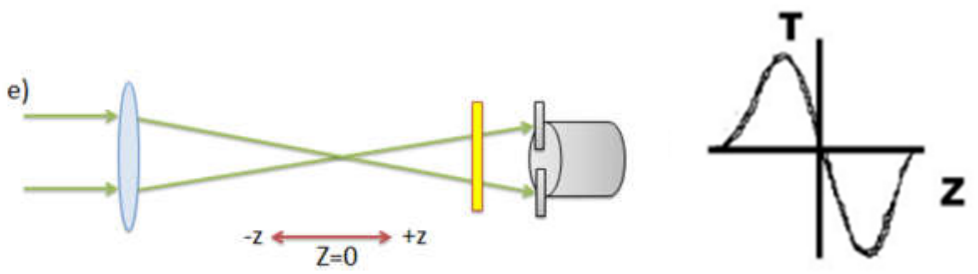

Figure 2-2: Illustration of the thermal-lens effect for a sample having a negative $d n / d T[48]$.

To obtain the characteristic of Z-scan curve (peak-valley/valley-peak) we normalize the transmittance at each position $\mathrm{z}$ and time $\mathrm{t}$ by equation [49].

$$
T_{N}(z, t)=\frac{I(z, t)}{I(z, 0)}
$$

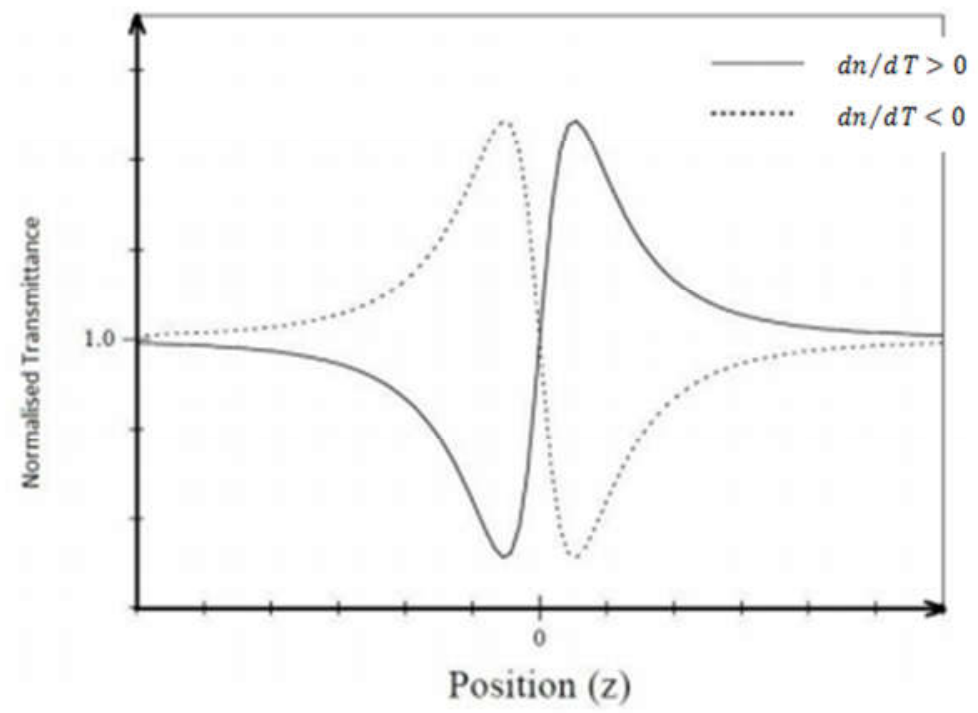

Figure 2-3: The Z-scan typical curve of the thermal-lens effect. Normalized Transmittance as a function of z-position for two samples with different signs of $d n / d T[50]$.

\section{2-1-2 Thermal-lens model}

The thermal-lens model was described well by Golden et al (1965) analyzing the changing in the transmittance of a non-focused Gaussian beam [51]. Whinnery and $\mathrm{Hu}$ continue this study on a focused Gaussian beam [52-53]. Thermal-lens effect due to a weakly absorbing medium induces rising in the local heating within the 
sample, generating a refraction-index gradient. The nonlocal dependence of the refractive index on the beam intensity causes the nonlinearity [54]. Thermal-lens phenomena are performed on the time scale of milliseconds [55]. There are two models to analyze the thermal effect and calculate the data obtained from the Z-scan experiment. The first one is known as a parabolic lens model and used by Golden, Whinnery and co-workers. This model assumes a parabolic approximation for the temperature distribution and that refractive index varies with increasing the temperature. The other model is called aberrant model, developed by Sheldon et al [56]. This model depends on the aberrant nature of the lens and uses the diffraction theory to study the intensity change at the center of the laser beam in the far field, and therefore, assumes the phase shift varies with increasing the temperature [57]. In 1984, Carter and Harris published a paper explaining the difference between these two models describing the thermal lens effect [20]. The parabolic-lens model will be used to investigate the thermo-optical properties of the low-density lipoprotein solutions (LDL).

When a Gaussian beam of mode $\mathrm{TEM}_{00}$ and beam radius $w$ propagates through a thin material, it causes an increase in the temperature, which is given by $[53,59]$

$$
\Delta T(r, T)=\frac{0.06 \alpha P}{\pi k}\left[E i\left(-\frac{2 r^{2}}{w^{2}}\right)-E i\left(-\frac{2 r^{2}}{8 D_{t h} t+w^{2}}\right)\right],
$$

where $\alpha$ is the light absorption coefficient, $P$ is beam power, $\kappa$ is the thermal conductivity of the sample, $r$ is the radial distance to the beam axis and $\operatorname{Ei}(x)$ is the exponential integral function. $D_{t h}$ is the thermal diffusivity and defined as:

$$
D_{t h}=\frac{\kappa}{\rho C_{p}}
$$

where $\rho$ is the mass density and $C_{p}$ is the specific heat capacity of the sample. 
The transmitted intensity is measured through an aperture in front of the photodetector. If this aperture is much smaller than the beam waist, a parabolic approximation to equation $(2,2)$ is used and gives:

$$
\Delta T(r, t)=\frac{0.06 \alpha P}{\pi k}\left[\ln \left(1+\frac{2 t}{t_{t h}}\right)-\frac{2 r^{2}}{w^{2}}\left(\frac{2 t}{2 t+t_{t h}}\right)\right]
$$

where $t_{t h}$ is the characteristic time of heat diffusion and is defined as:

$$
t_{t h}=\frac{w^{2}}{4 D_{t h}}
$$

Due to the sample light incident absorption, the temperature increases and causes a change in the density of the sample, hence a change in the refractive index. This variation of the refractive index is proportional to $\Delta T$ and is written as:

$$
n(r, t)=n_{0}+\frac{d n}{d T} \Delta T(r, t)
$$

where $n_{0}$ is the linear refractive index and $d n / d T$ is the thermo-optic coefficient, which gives the nonlinearity.

We consider the refractive-index changes with the beam radius as quadratic, and the thermal lens will act as an ideal thin lens with the focal length given by [55, 59]:

$$
\frac{1}{f(t)}=-\left.l_{e f f} \frac{d n}{d T} \frac{d^{2}}{d r^{2}} \Delta T(r, t)\right|_{r \rightarrow \infty},
$$

where $l_{e f f}$ is the effective propagation length of the sample and given by:

$$
l_{\text {eff }}=\frac{1-e^{-\alpha L}}{\alpha}
$$

$\mathrm{L}$ is the sample thickness.

The normalized transmittance for each z-position and time $t$ is writing as $[49,59]$ :

$$
T_{N}(z)=\frac{I(z, t)}{I(z, t=0)}=\left[1-2 x\left(\frac{\theta}{1+x^{2}} \frac{2 t}{2 t+t_{t h}}\right)+\left(1+x^{2}\right)\left(\frac{\theta}{1+x^{2}} \frac{2 t}{2 t+t_{t h}}\right)\right]^{-1},
$$
where $x$ is the dimensionless sample position and written as $x=z / z_{0}$. 
$z_{0}$ is the Rayleigh length and given as: $z_{0}=\frac{\pi w_{0}^{2}}{\lambda}, \lambda$ is the light wavelength and $w_{0}$ the beam waist at the focal position $(\mathrm{z}=0)$.

$\theta$ is known as the amplitude of the Z-scan characteristic curve, which depends on several parameters and given by:

$$
\theta=\frac{0.24 \alpha P l_{e f f}}{k \lambda}\left(-\frac{d n}{d T}\right)
$$

The amplitude of thermal lens can be calculated also from the difference between the transmittance peak and valley. Figure $2-4$ shows the peak-valley difference $\Delta_{\mathrm{p}-\mathrm{v}}^{\mathrm{TLM}}$, which is written as [49]:

$$
\Delta_{p-v}^{\mathrm{TLM}} \approx 2|\theta| .
$$

We analyze the data obtained from the Z-scan experiment through a parabolic thermal-lens model then we can determine the amplitude of the Z-scan characteristic curve. This amplitude depends on several parameters such as laser beam power, linear absorption coefficient, thermo-optical coefficient and thermal conductivity. Therefore we study the thermal optical properties on LDL solutions by equation (2.7).

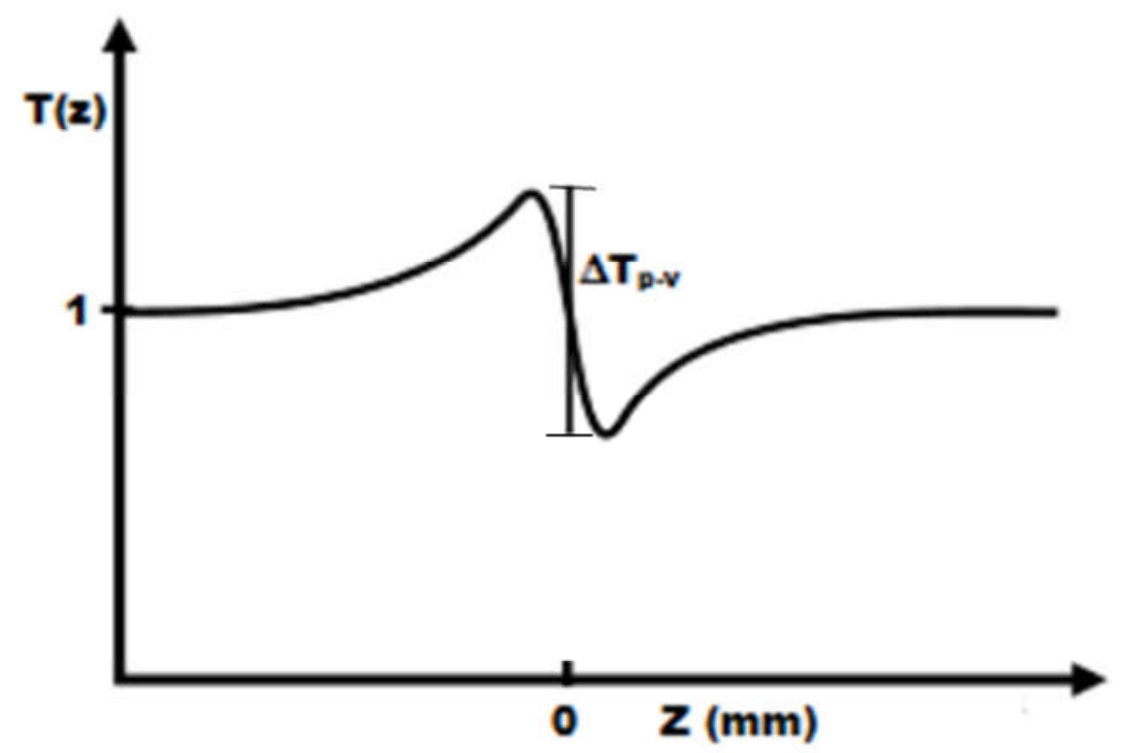

Figure 2-4: Graph illustrating the meaning of $\Delta \mathrm{T}_{p-v}$ [48] 


\section{2-1-3 Experimental setup}

The setup of Z-scan experiment is illustrated in Figure 2-5.The light source used in the experiment was from a Nd: YVO4 $\mathrm{cw}$ laser (continuous wave) with a Gaussian profile (TEM00 mode) 532nm wavelength (green light) and radius w. The beam power used for LDL samples was $(100 \pm 5) \mathrm{mW}$. The beam passes through two converging lenses (L1, L2). Each lens has diameter of $50.8 \mathrm{~mm}$ and a focal length of $100 \mathrm{~mm}$ for $\mathrm{L} 1$ and $80 \mathrm{~mm}$ for L2 and are used to focolize the beam.

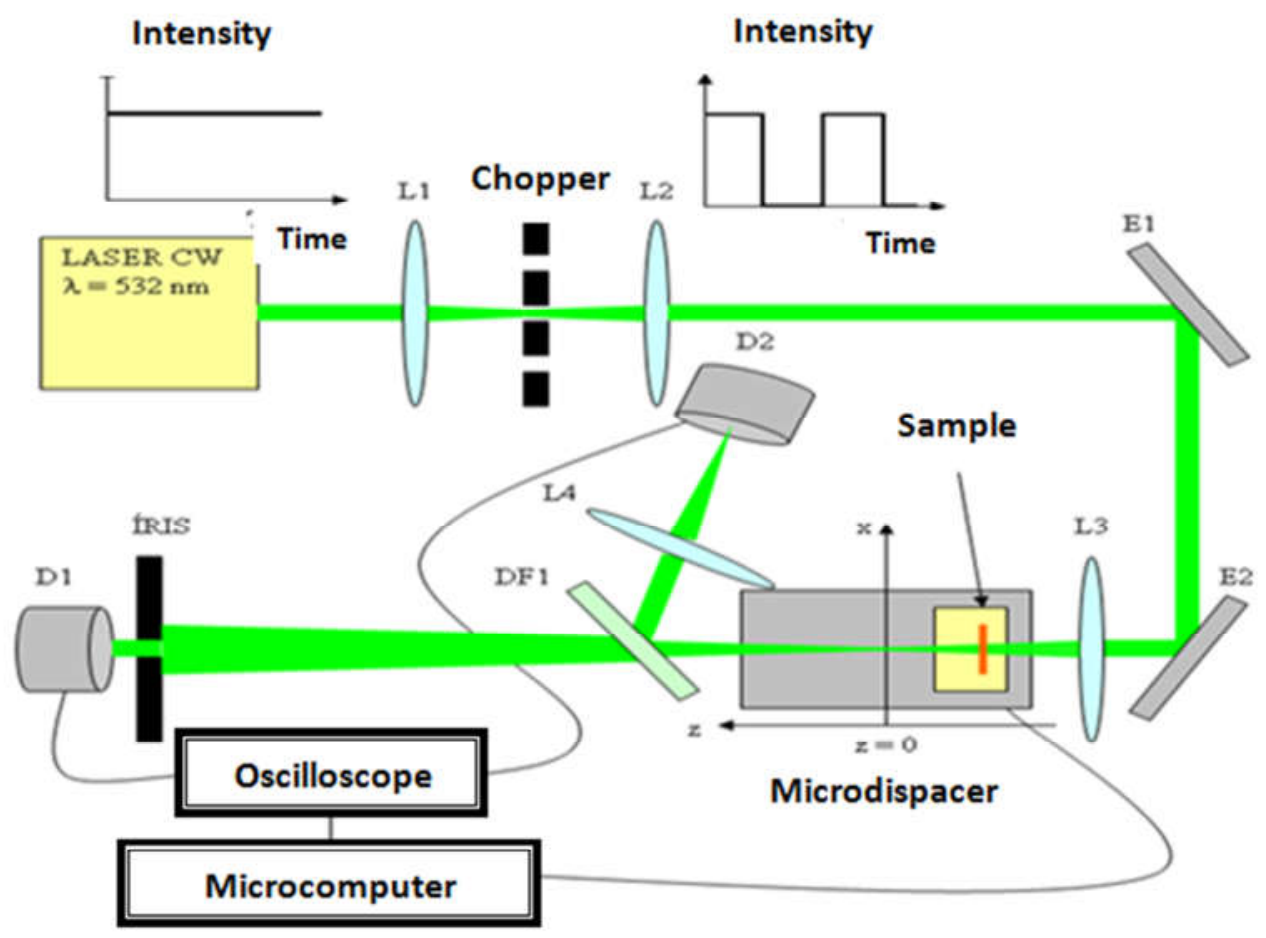

Figure 2-5: Experimental setup of Z-scan technique [60].

A mechanical chopper is placed between the lenses (L1, L2) at the focal point. The chopper is used to modulate the beam periodically, giving square pulses in the time scale of milliseconds. Knowing how to choose the time interval $\Delta T$ is important to avoid some undesirable effect such as a cumulative effect. This effect appears when the time interval between pulses is too short compared to thermal characteristic time 
$\left(\Delta T \ll t_{t h}\right)$ because there is not enough time between two pulses to completely relax the system, therefore the thermal lens does not reach the stationary state. Another effect could also appear if the time interval is too long compared to thermal characteristic time $\left(\Delta T \gg t_{t h}\right)$, such as the Soret effect [59]. For LDL samples we applied $\Delta T \approx 30 \mathrm{~ms}$, which is provided by a chopper of frequency of $16 \mathrm{~Hz}$.

The beam after lens L2 is reflected by two mirrors (E1, E2) and has a diameter of $25.4 \mathrm{~mm}$. These mirrors are used to align the laser beam to another lens (L3) with a diameter 50,8 $\mathrm{mm}$ and focal length of $125 \mathrm{~mm}$. This is known as the Z-scan lens. This lens focuses the beam at position $z=0$. The sample is placed on a microdisplacer, which moves it through the beam focus in the z-direction and at different positions. This microdisplacer is controlled through the microcomputer by the acquisition program. The sample is also placed inside a temperature control device, which adjusts the sample temperature. The temperature of the LDL samples was fixed at $37^{\circ} \mathrm{C}$ because it is the temperature of LDL in the human body.

After the sample, a beam splitter is inserted into the beam path to divide the beam in two directions. Part of the beam is sent in the first direction to the photodetector of silicon (D1). The detector is positioned far from the focal point of L3 and sensitive to the intensity change in the center of the laser beam. The other part of the beam is aligned to another photodetector. The two detectors are connected to oscilloscope (Tektronix), which reads the signal and send it to the microcomputer to process the data by acquisition program.

\section{Z-scan, data acquisition:}

Z-scan data are obtained through the acquisition software "zscan_chopper". The program also controls the microdisplacer, and it was developed by the Group of Complex Fluids at the Institute of Physics in USP. "Zscan_chopper" program is 
connected with the oscilloscope and then collects the same data of the voltage value as a function of time from channel 1 , which is related with the photodetector D1 and from channel 2, which is related to the second photodetector D2. Through the window shown in Figure 2-6 we can control several parameters related to the microdisplacer, such as the total displacement through which the sample moves, that was $50 \mathrm{~mm}$ for LDL samples. The $\mathrm{z}$ interval between the positions was $1 \mathrm{~mm}$, the number of measurements at each position was 10 times, and the waiting time between the displacements was $1 \mathrm{~s}$.

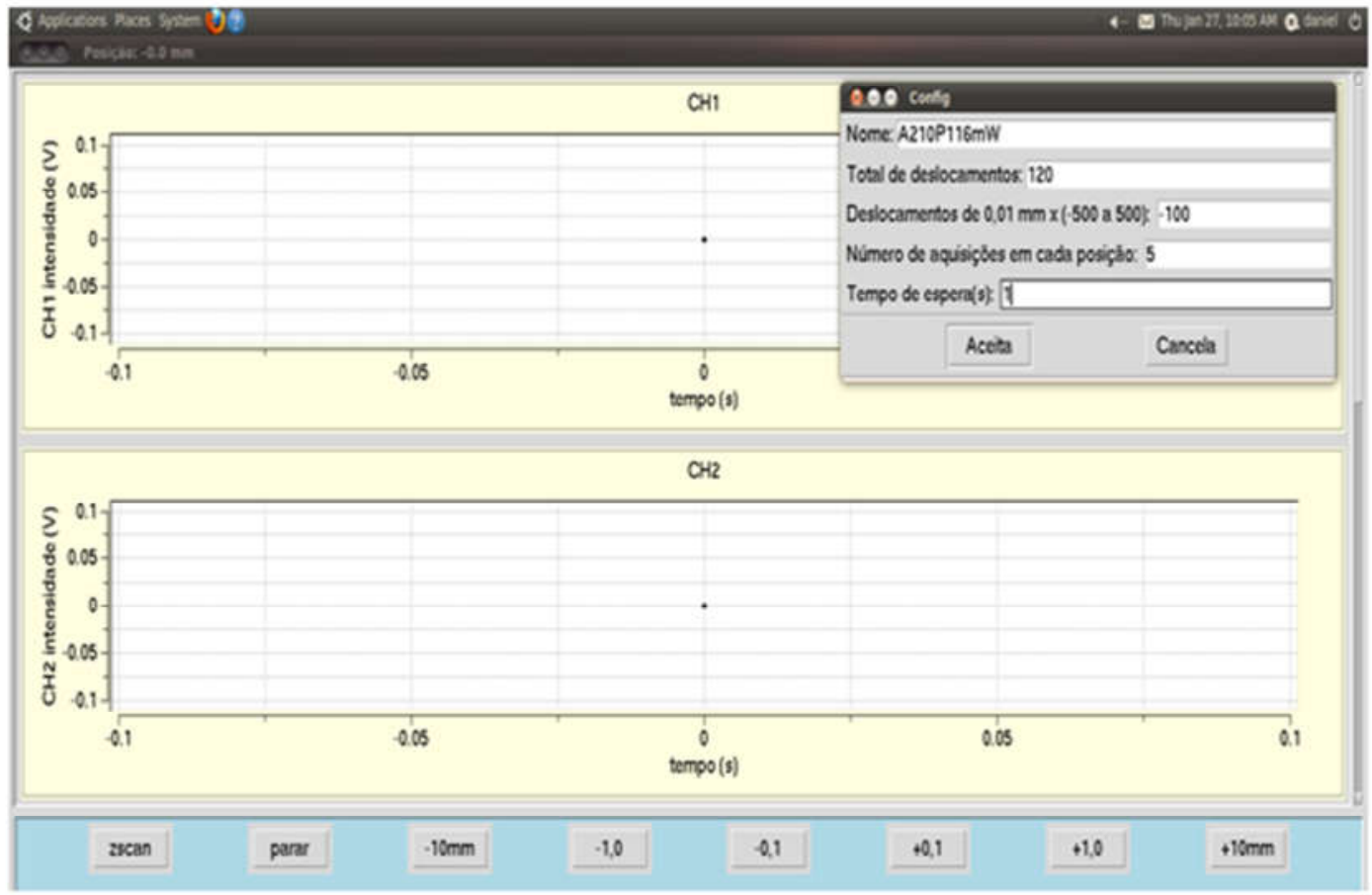

Figure 2-6: Configuration of the Z-scan arrangement through the zscan_chopper software [60]. 


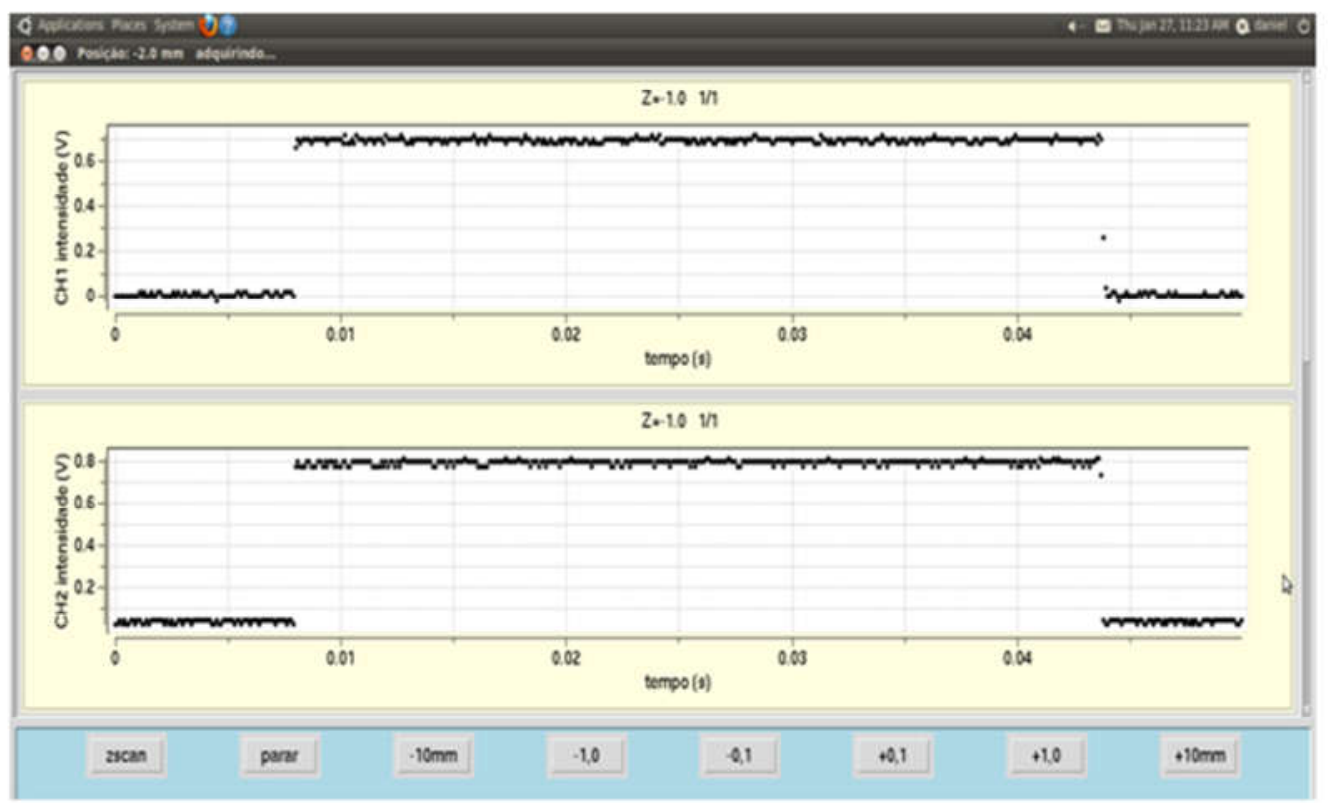

Figure 2-7: zscan_chopper_new software with the data collected from the oscilloscope [60].

Figure 2-7 shows a window of the "zscan_chopper" program with data obtained from the oscilloscope. It also presents, for a specific position, the square electrical signal as a function of time, for both channel 1 and channel 2 .

During the measurement performed, the thermal-lens effect appears clearly through the square electrical signal where we could see the time evolution of the light transmittance. The time evolution of the light transmittance is defined as the light transmittance as a function of time at a fixed position. For thermal-lens effect, all time-dependent curves start at the same transmittance value. Figure 2-8 shows the pulse recorded on the oscilloscope screen. When the sample is placed at the focus, the transmitted intensity has the same value, then when the sample moves towards the beam focus the transmitted intensity changes significantly at the beginning of the pulse. Far from the focus, the transmitted intensity also returns to the same value. 


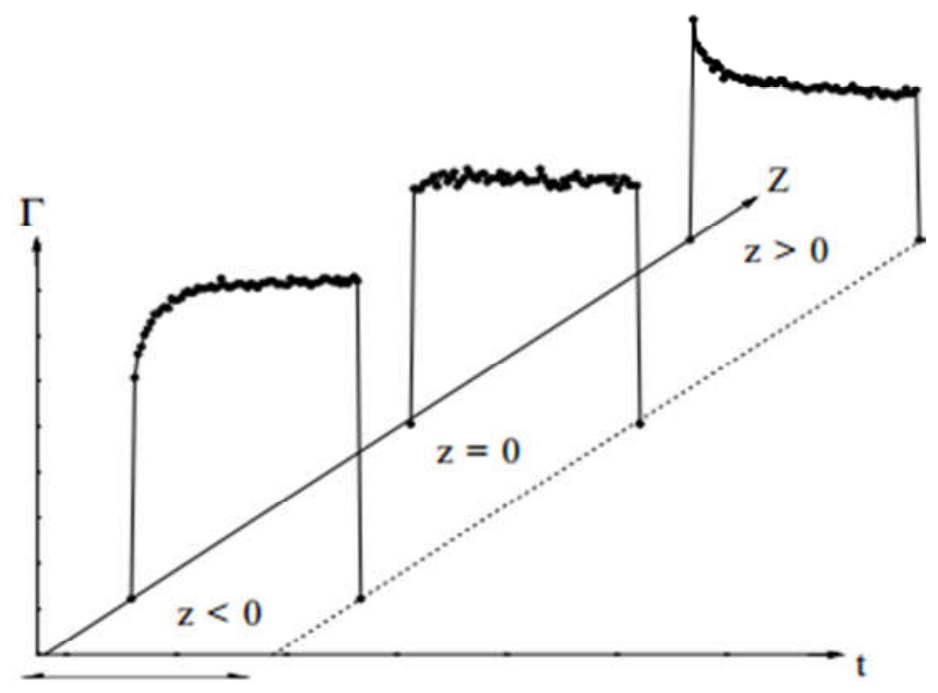

Figure 2-8: Typical oscilloscope's records of the transmittance, as a function of time, of a sample with negative $d n / d T$ [50].

After the Z-scan measurement is finished all the data is stored at "zscan_chopper" program. Through the program, we can do the normalization for the transmittance data to obtain the Z-scan characteristic curve (peak-valley/valley-peak) according to equation (2.1) then save it in a file. This file contains the data of position, normalized transmittance $T_{N}$, and the standard deviation. The file can be opened in another program like QtiPlot to process and fit the data with models.

\section{2-2 Linear absorbance experiment}

\section{2-2-1 Measurement of the linear absorption coefficient}

When the light interacts with a material some of the light can be absorbed, scattered and transmitted, as shown in Figure 2-9. The transmission of the material is defined as the ratio of the initial intensity $\left(I_{0}\right)$ to the transmitted intensity $\left(I_{T_{r}}\right)$ and given by the equation [61]: 


$$
T_{r}=\frac{I_{T_{r}}}{I_{0}}=e^{-\alpha L},
$$

where $\alpha$ is known as the linear absorption coefficient and $\mathrm{L}$ is the sample thickness.

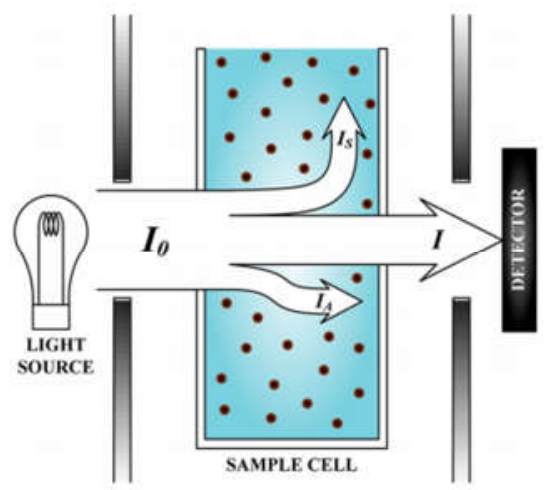

Figure 2-9: Illustrative scheme of the transmission when the light passes through a material.

By knowing the transmission of the material, the absorbance is defined by the equation:

$$
A=\log \left(\frac{1}{T_{r}}\right)
$$

where the absorbance is proportional to the concentration of the material. By using equation (2.9) and (2.10) the absorption coefficient is calculated as:

$$
\alpha=\frac{A \ln (10)}{L} .
$$

For LDL nanoparticles, they present a significant scattering beside the absorbance and the transmission, therefore, couldn't be neglected. This scattering is related to Rayleigh scattering, which is determined by the equation [61]:

$$
\operatorname{Sca}(\lambda)=a * \lambda^{-4}+b
$$

where $\mathrm{a}, \mathrm{b}$ are constants and $\lambda$ is the wavelength of the light. 


\section{2-2-2 Experimental setup}

UV-visible spectrophotometer measures the absorbance of LDL samples. The light source consists of a deuterium lamp and halogen lamp. The deuterium lamp emits electromagnetic radiation with a wavelength from $200 \mathrm{~nm}$ to $800 \mathrm{~nm}$ and the halogen lamp emits electromagnetic radiation with wavelengths from $500 \mathrm{~nm}$ to 1100 $\mathrm{nm}$. The light comes from the source to the sample then to two spectrometers through optical fibers. The spectrometer measures the transmitted intensity then converts it into an electrical signal and send it to an acquisition program called "Spectra Suite" (Ocean Optics) in the microcomputer.

The spectrophotometer is calibrated with an empty reference cuvette through measuring the intensity when no radiation passes the cuvette $\left(I_{z}\right)$, and the intensity after emitting the radiation from the source to the empty cuvette $\left(I_{R}\right)$. Then we insert a cuvette filled with a sample solution to measure the intensity of radiation transmitted through the sample $\left(I_{A}\right)$. Spectra Suite processes this data and calculates the absorbance relative to a reference material by using the following equation:

$$
A(\lambda)=-\log _{10}\left(\frac{I_{A}(\lambda)-I_{Z}(\lambda)}{I_{R}(\lambda)-I_{Z}(\lambda)}\right) .
$$

The results of the UV-visible spectroscopy measurement are shown in the absorbance spectra in the Spectra Suite program. But actually, for LDL nanoparticles, the absorbance spectra refer to the extinction spectra, which is the sum of both scattering and absorbance, because the spectrometer measures only the light transmitted, therefore we could not differentiate between the absorbance and the scattering. To obtain the absorbance value we subtract the scattering, which is calculated by equation (2.12) from the extinction spectra. Then the linear absorption 
coefficient calculated by equation (2.11) at the same wavelength $\lambda=532 \mathrm{~nm}$ is used for the Z-scan experiment. The measurements are performed at temperature $37^{\circ} \mathrm{C}$. The thickness of the cuvettes used is $10 \mathrm{~mm}$.

\section{2-3 Measurement of the thermo-optic coefficient}

To obtain the thermo-optical coefficient $(d n / d T)$, the refractive index of LDL samples is measured at a different temperature through an ATAGO 5000i refractometer. The accuracy of measurements is about $0.1^{\circ} \mathrm{C}$ in the temperature and

$2 \times 10^{-5} \mathrm{nD}$ in the refractive index. The temperature used varies between 26 and $46^{\circ} \mathrm{C}$. The data obtained is analyzed to determine the linear slope, which provides the thermo-optical coefficient value in $T^{-1}$ unit.

\section{2-4 Dynamic light scattering technique}

Dynamic light scattering (DLS), also known as photon correlation spectroscopy, is an easy and quick way to measure the size distribution of nanoparticles in solution, even though it is a low-resolution technique. Basically, when a sample of a colloidal dispersion, which contains a large number of particles, is illuminated by a laser beam, the intensity fluctuations over time of the scattered light from all illuminated particles are detected at a fixed scattering angle $\theta$ by a photodetector $[62,63]$. Figure 2-10 shows the basic DLS experimental geometry. 


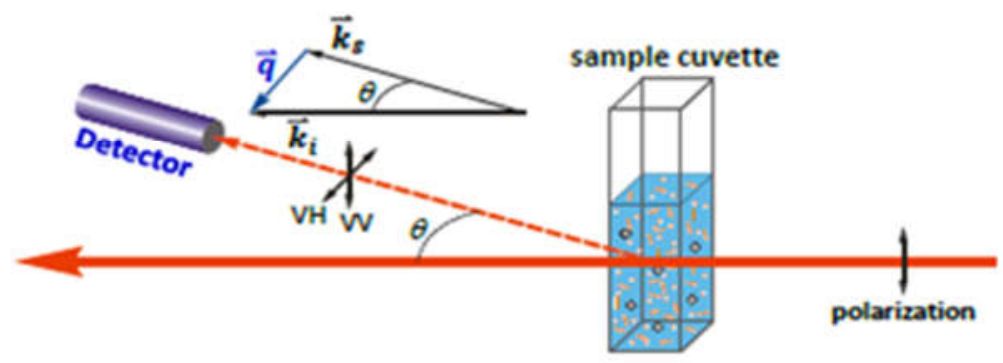

Figure 2-10: sketch of the DLS setup.

As is well known, at a given temperature, the particles in a fluid undergo Brownian motion. Small particles move faster than larger particles. This diffusional motion is the main cause of the temporal fluctuations in the intensity of the scattered light, and consequently the source of information about the particle structure, provided that a suitable model relating structure and time scale of the movement (diffusion) of the particles exists.

The measurements of these temporal fluctuations are made through autocorrelation of the detected scattered intensity signal, at a given angle $\theta$. The decay time of fluctuations depends on the diffusion constant and the size of particles. The small particles move rapidly, therefore it takes a lower decay time while the large particles move slowly, therefore it takes a higher decay time. The fluctuating signal is processed through autocorrelation function $C(t)$, which is given by the following expression for the monodisperse case [62,63]:

$$
C(t)=A e^{-2 \Gamma t}+B
$$

where $\mathrm{t}$ is the delay time, $\mathrm{A}$ and $\mathrm{B}$ are constants and $\Gamma$ is the decay constant related to the relaxation of the fluctuation and given as

$$
\Gamma=D q^{2}
$$


where $\mathrm{D}$ is the diffusion coefficient and $\mathrm{q}$ is the modulus of scattering vector that depends on the scattering angle $\theta$ through the equation:

$$
q=\frac{2 \pi n}{\lambda_{0}} 2 \sin \left(\frac{\theta}{2}\right)
$$

where $\mathrm{n}$ is the average refractive index of the medium at a given temperature $\mathrm{T}$, and $\lambda_{0}$ is the wavelength of the laser light in vacuum.

From the equations 2.14-2.16, the diffusion coefficient could be obtained from a proper analysis of the measured autocorrelation function. If it is known for a given particle, and also making the hypothesis of spherical, non-interacting nanoparticle, the mean size of these nanoparticle can be determined directly from the Stokes-Einstein equation,

$$
r=\frac{K_{B} T}{6 \pi \eta(t) D}
$$

where $\mathrm{r}$ is the so called hydrodynamic radius of the nanoparticle, $K_{B}$ is Boltzmann's constant, $\mathrm{T}$ is the sample's equilibrium temperature, $\eta(\mathrm{t})$ is the viscosity of the liquid. The Stokes-Einstein equation is an example of a suitable model relating structure and diffusion of particles in suspension, assuming the hypotheses described above.

The LDL nanoparticles are spherical in shape to a good approximation [64]. And in practice the non-interacting regime for a nanoparticle solution is achieved by working in an appropriate concentration range, where the average distance between the particles is higher than the characteristic distances of the interactions between the particles. If necessary, the solution is diluted by adding solvent, taking care to keep the chemical stability of the sample and also to avoid coalescence and/or aggregation of the particles. So that, the Stokes-Einstein equation is a suitable model for the diffusion of LDL particles in solution $[65,66]$.

DLS is an ensemble-average technique in opposition to single particles techniques, like transmission electron microscopy that analyzes the particles individually, one at a time. 


\section{2-4-1 Experimental setup}

The DLS measurements were made using a 90plus Particle Size Analyzer from Brookhaven Instruments (USA). In this equipment, the samples are illuminated by a laser beam with wavelength of $657 \mathrm{~nm}$ and power of $35 \mathrm{~mW}$. The intensity fluctuations of the scattered light are registered by a photodetector placed at an angle of $90^{\circ}$ relative to the incident beam direction. The technique is suitable to measure sizes of particles from $2 \mathrm{~nm}$ to $3 \mu m$.

For our LDL samples, the measurements were performed at $37^{\circ} \mathrm{C}$ for 5 minutes, divided in measurements of 1 minute for statistical analysis and evaluation of the data quality. The measured data were analyzed by Brookhaven Instruments Dynamic Light Scattering Software. Among other methods of analysis [62], the Non-Negatively constrained Least Squares (NNLS) method was the one that resulted in better and more stable fit of the experimental data. So that, it was chosen to estimate the particlesize distribution histogram weighted by number, volume, and intensity of scattered light for each sample. From the number-averaged size distribution, an average hydrodynamic radius and standard deviation of the distribution were calculated. 


\section{3- Samples}

This study carried out on 24 diabetes mellitus type 2 (DM2) patients with generalized chronic periodontitis (group 2) and 24 DM2 patients with gingivitis (group 1).

The group of DM2 patients with periodontitis received a periodontal treatment and analyzed after 6 months. With respect to time, the samples were divided into basal time (time 0) and 6 months after treatment (time 6). Therefore, the samples were distributed according to the time (time 0 and time 6 ) and the presence of periodontitis (group 1 and group 2).

These patients were collected at the Science and Technology Institute (ICT) - School of Dentistry of São José dos Campos (FOSJC-UNESP), in São José dos Campos - SP - Brazil.

To determine the sample size, a confidence interval of $95 \%$ and a $\beta$-error of $20 \%$ (power of $80 \%$ ) were used to detect a difference of 0.12 units of modified LDL between the groups [30].

\section{3-1 Selection Criteria}

The 48 patients analyzed showed the pre-established inclusion and exclusion criteria for this study. 


\section{3-1-1 Inclusion Criteria}

- Individuals older than 35 years with DM2 diagnosed for more than five years and with glycated hemoglobin A1c (HbAlc) levels controlled (between 7\% and $11 \%)$;

- To be diagnosed with generalized chronic periodontitis: to present loss of clinical interproximal insertion $>3 \mathrm{~mm}$ in 2 non-adjacent teeth and loss of interproximal clinical insertion $\geq 5 \mathrm{~mm}$ in $30 \%$ or more of the teeth present [67];

- To have present at least 20 teeth;

- To agree to participate in the study and sign the informed consent form (Resolution CNS 466/12 and the Professional Ethical Code - 179/93).

\section{3-1-2 Exclusion Criteria}

- Patients with a history of hypertension or nephropathies;

- Patients with cancer, gastrointestinal disorders, skin diseases, pregnancy, lactation, smoking, arthritis and lupus;

- To have undergone periodontal treatment in the last 12 months;

- To have made use of an antioxidant, anti-inflammatory, or antibiotic supplements within the previous 3 months;

- To have changed the medication for glycemic control in the last 3 months;

- To present dental elements with pulpal or periapical inflammation;

- To present any other inflammatory diseases. 


\section{3-2 Separation of lipoprotein}

Blood samples were collected from each individual after fast of 12-15 hours. The collection was conducted by qualified professional and in a reserved place, equipped exclusively for this purpose. All material needed for the collection of blood was used for the first time and had its disposal done within the laboratory safety standards. All samples were used exclusively for the purposes described in this study. The blood was collected in vacutainer tubes containing Ethylene Diamine Tetraacetic Acid-EDTA $(1,0 \mathrm{mg} / \mathrm{mL})(\mathrm{BD} \AA$, Brazil), used as anticoagulant and antioxidant, kept on ice and protected from light until the plasma was obtained (by centrifugation at $3000 \mathrm{rpm}, 10$ $\left.\min , 4^{\circ} \mathrm{C}\right)$. Plasma separation was done on the same day of the blood collection. After, the plasma samples were stored at $-80^{\circ} \mathrm{C}$. The phase of blood collection, plasma separation and storage was done in ICT. The step of obtaining the LDL from the plasma was performed in Laboratory of the Institute of Physics of USP. The transport of the samples showed kept on ice and protected from light until final destination (IF/USP).

The following were added to the plasma: benzamidine $(2 \mathrm{mM})$, gentamicin $(0.5 \%)$, chloramphenicol (0.25\%), phenyl-methyl-sulfonyl-fluoride (PMSF) (0.5 mM), and $\operatorname{aprotinin}(0.1 \mathrm{U} / \mathrm{mL})$.

From plasma, very low density lipoprotein (VLDL) $(1.006<\mathrm{d}<1.019 \mathrm{~g} / \mathrm{mL})$ and low density lipoprotein $(\mathrm{LDL})(1.020<\mathrm{d}<1.063 \mathrm{~g} / \mathrm{mL})$ were isolated by preparative sequential ultracentrifugation $\left(40,000 \mathrm{rpm}, 18\right.$ hours at $\left.4^{\circ} \mathrm{C}\right)[68]$ using a fixed-angle

rotor (Hitachi ${ }^{\circledR}$ Himac CP 70MX, Tokyo, Japan). The VLDL samples were discarded. The density cut-off point for to isolate LDL was $1.063 \mathrm{~g} / \mathrm{mL}$. 
All reagents were purchased from Sigma Chemical ${ }^{\circledR}$ (St. Louis, MO, USA). All biological waste not used in the described experiments was discarded according to the Laboratory Safety Standards.

\section{3-3 Biochemistry analyses}

\section{3-3-1 Protein concentration}

Protein concentrations in LDL were determined by using the Bicinchoninic Acid (BCA) method, PierceTM BCA Protein Assay kit (Thermo Fisher Scientific ${ }^{\circledR}$, MA, USA) with bovine serum albumin (BSA) as standard [69].

\section{3-3-2 Glycolaldehyde concentration}

Long-term incubation in vivo of the LDL with glucose, especially in patients with diabetes, leads to the formation of advanced glycation end products (AGE). The AGE concentration in LDL samples was determined in sub-sample using a Cell Biolabs' OxiSelect $^{\mathrm{TM}}$ AGE Competitive ELISA Kit (Cell Biolabs ${ }^{\circledR}$, San Diego, CA, USA).

Measurements were performed in duplicate and the results were averaged, according to manufacturer's instructions. The ratio of AGE concentration to protein level was expressed in micrograms of AGE per milligram of protein $(\mu \mathrm{g} / \mathrm{mg})$.

\section{3-4 Statistical analysis}

Quantitative variables were evaluated using the Statistical Package for the Social Sciences (SPSS) version 16.0 [70].

For the determination of the tests we considered the type of distribution of the variables, using the Kolmogorov-Smirnov test $(p>0.05)$. For the variables with 
normal distribution we used a parametric test and for the other variables, a nonparametric test. The significance level considered for all tests was $\mathrm{p}<0.05$. 


\section{4- Experimental results and discussion}

\section{4-1 Z-scan}

The Z-scan experimental setup as illustrated in Figure 2-5 is used to study the nonlinear optical response of the low-density lipoprotein (LDL) from two groups of patients with diabetes (group 1) and patients with diabetes and periodontitis (group 2). The degree of modification of LDL particles in the solution is investigated by the amplitude of Z-scan typical curve (peak to valley) where the higher amplitude value $(\theta)$ corresponds to the less modified degree, as Gomes et al and Santos et al presented in their study $[67,37]$. The amplitude value depends also on the sample LDL concentration [37]. In this study each sample has a different LDL concentration. To compare the results it is necessary to normalize the amplitude values of Z-scan measurements for all of them to have the same concentration by the equation:

$$
\theta_{n(\text { new })}=\frac{C_{\max }}{C_{n}} \theta_{n(\text { old })},
$$

where $C_{\max }$ is the maximum concentration of the samples and $C_{n}$ is the concentration of sample n.

Figure 4-1 shows the typical Z-scan curves for some LDL samples from group 1 and group 2 in the beginning (time 0) and after 6 months (time 6). In Figure 4-1(a), a correspondence is observed between the experimental data and the fitting with the theoretical model of equation 2.6. Figure 4-1(b) shows that the amplitude of the ZScan curve of patient with diabetes and periodontitis is lower than of the patient with diabetes and gingivitis, and Figure 4-1(c) shows that the amplitude of the Z-Scan signal of a patient with diabetes and periodontitis increased after 6 months.

To study the amplitude values $(\theta)$ that is obtained by equation (2.6) for the two groups of patients we will present the results in the box plot. 
a-

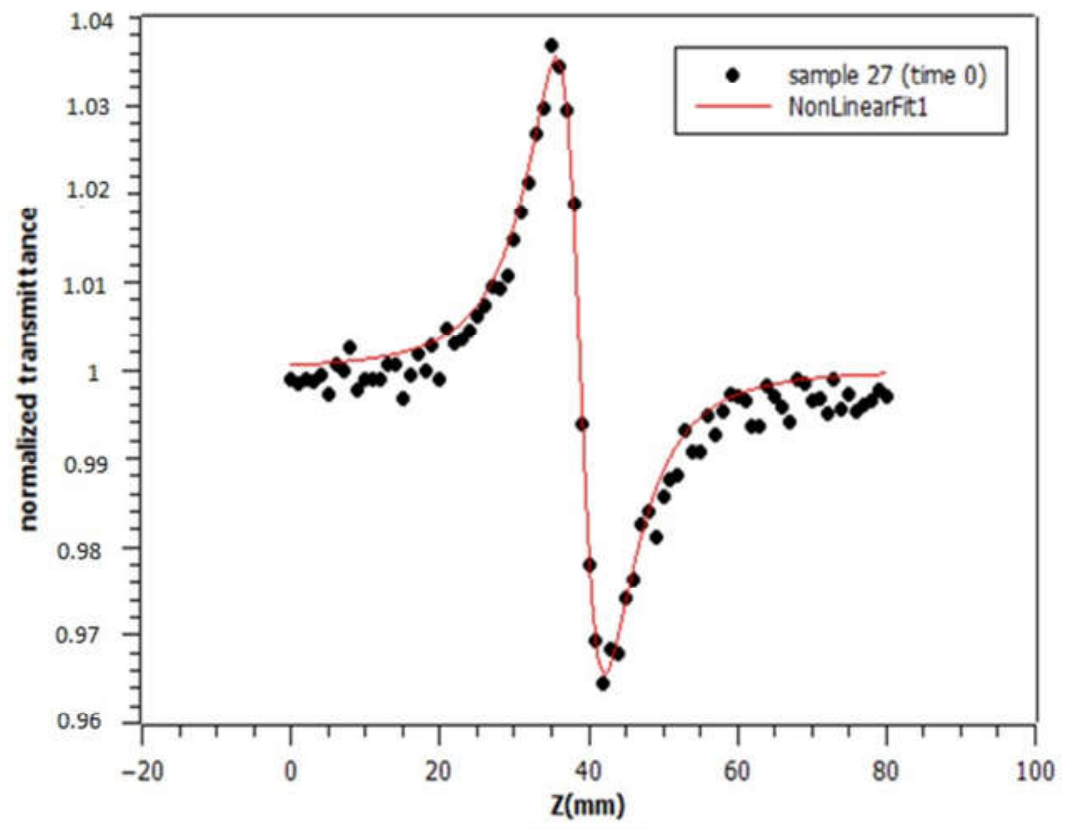

b-

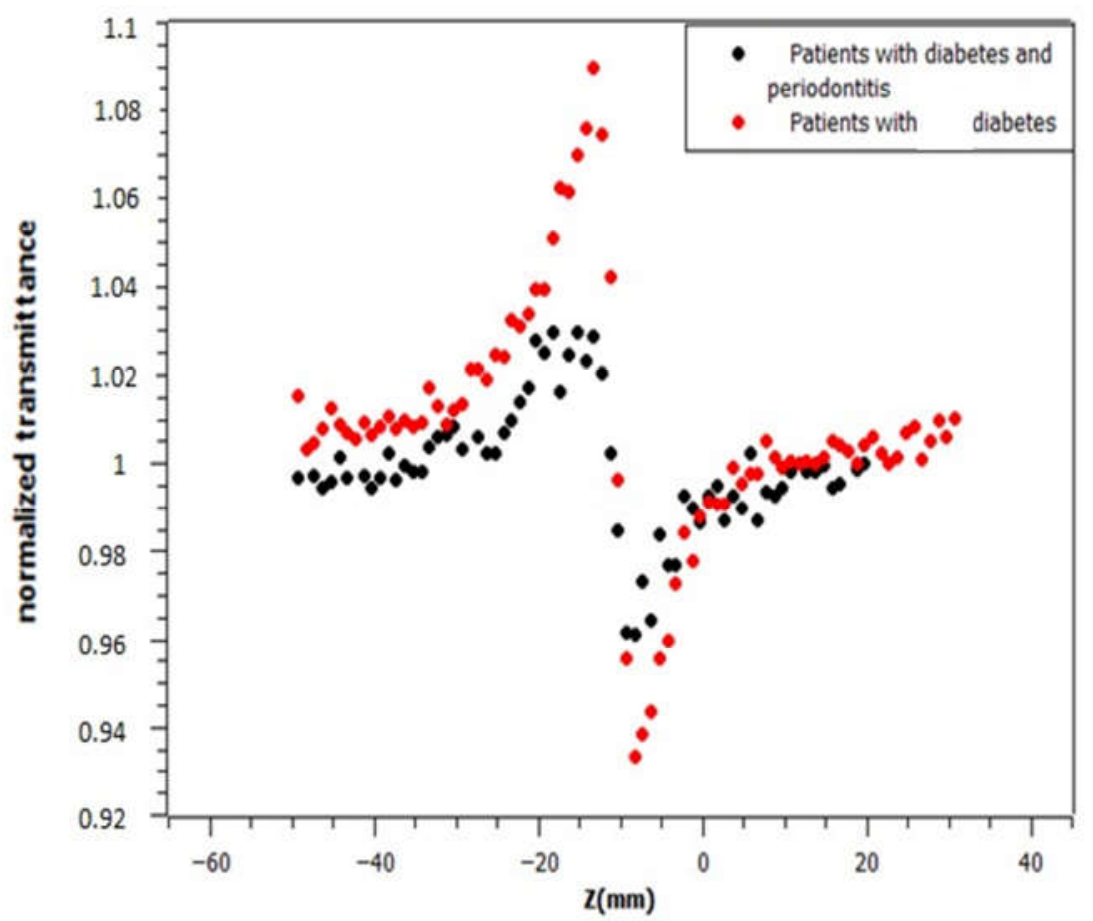


c-

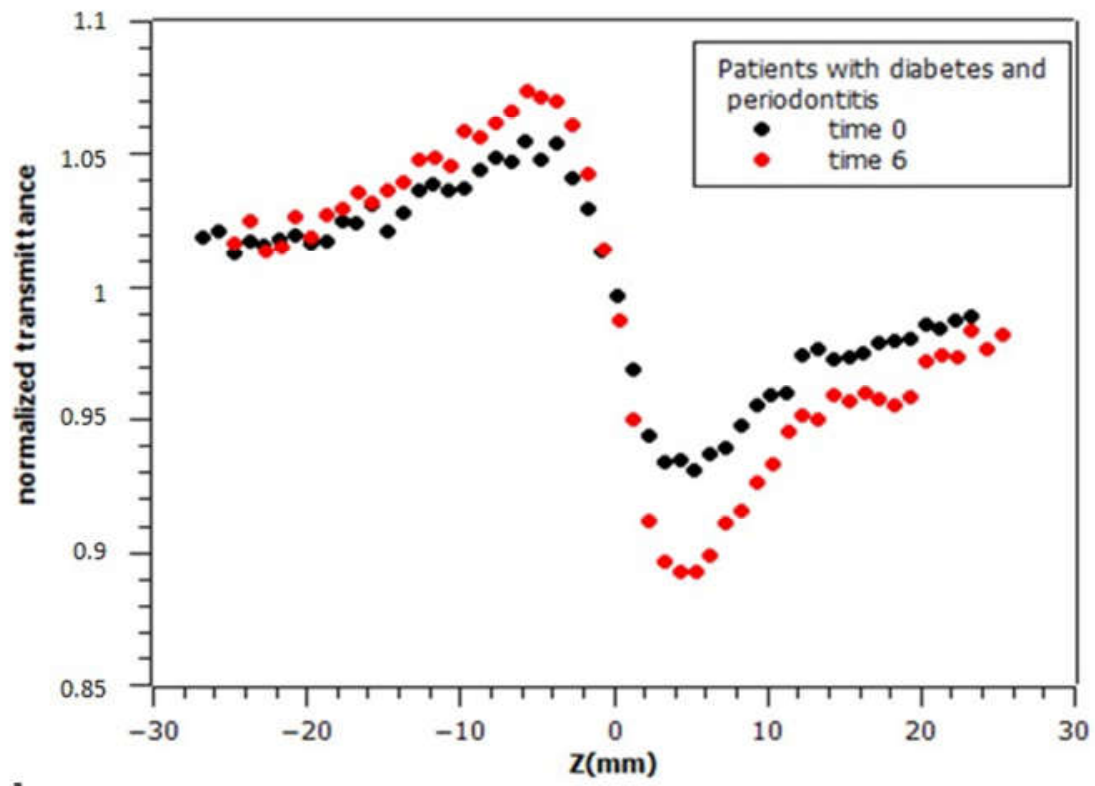

Figure 4-1: Comparison of Z-Scan typical curve between some patients. (a) experimental data and theoretical fitting. (b) patients with diabetes and periodontitis versus patients with only diabetes. (c) evolution of the z-scan signal after six months.

In the beginning (time 0 ), the amplitude values $(\theta)$ of the 24 patients from group 1 and the 24 patients from group 2 are presented in the box plot shown in the Figure 42. The results show that the mean value of $\theta$ from the patients of group 1 is higher than group 2. This means that LDL particles of the patients with diabetes and periodontitis are more modified than those from the patients with diabetes and gingivitis. The higher modification of LDL corresponds to the lower amplitude (peak to valley) and this result is the same as what other studies showed. 


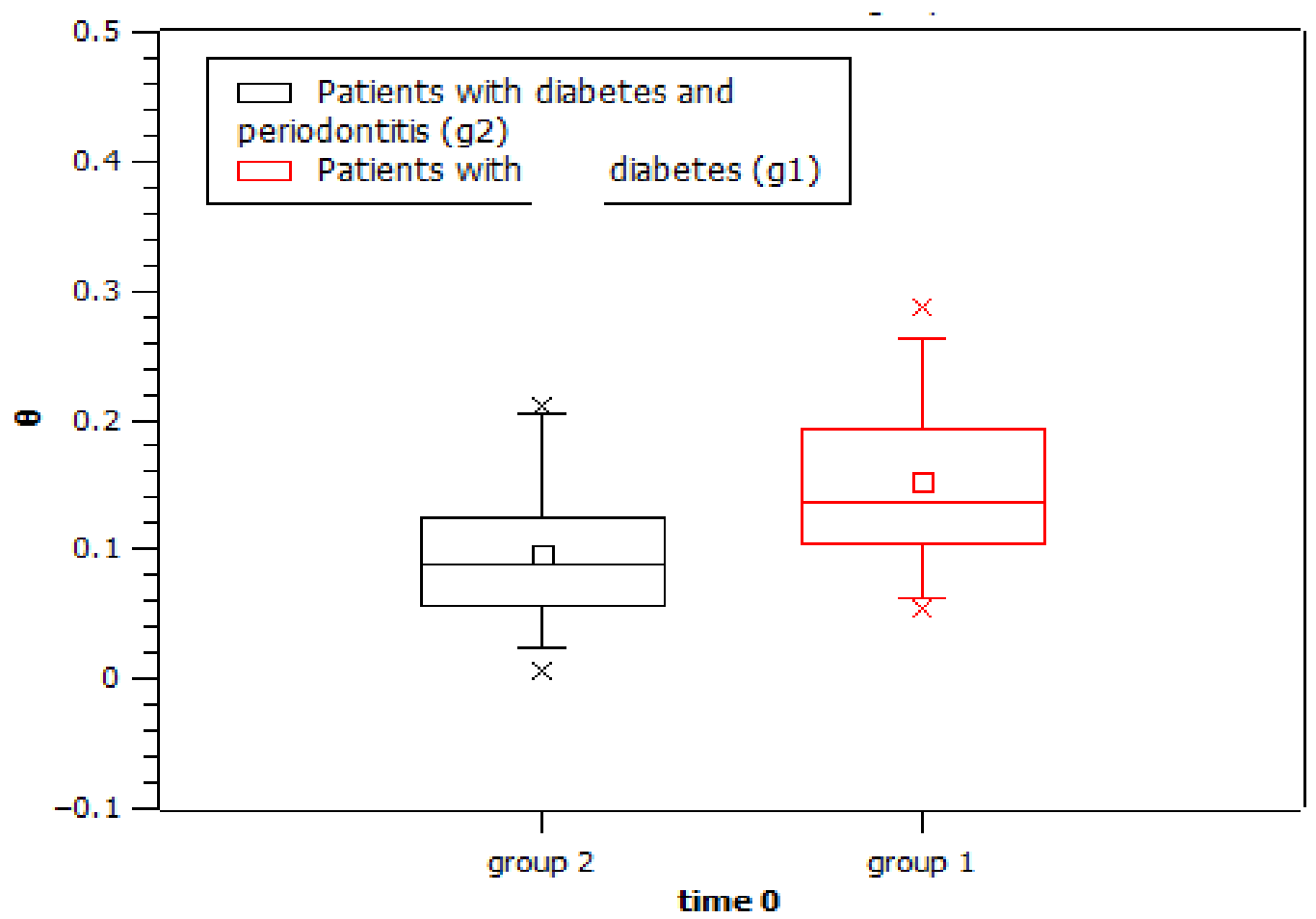

Figure 4-2: Box plot of the $\theta$ values of all the patient from group 1 and group 2 in the beginning (time 0$)(p=0.002)$.

Figure 4-3 shows the box plot of the amplitude values $(\theta)$ of all the patients from group 2 before and after 6 months of the periodontal treatment. For group 2 the results show that the mean value of $\theta$ after 6 months periodontal treatment is higher than that before treatment. The degree of modification of LDL particles was showed to be reduced after the treatment. Therefore, the patients of group 2 have less modified LDL particles after the treatment than before.

The Z-scan is performed also for LDL samples from group 1 after 6 months without any treatment. Figure 4-4 shows the comparison of $\theta$ values between the two groups after 6 months. The mean value of $\theta$ from the patients of group 1 is still higher than the patients of group 2 but with a lower percentage than the beginning.

Figure 4-5 shows the amplitude values $(\theta)$ of all the patients from group 1 in the beginning and after 6 months. For group 1, the results show that there is no significant change in the mean value of $\theta$ before and after 6 months. 


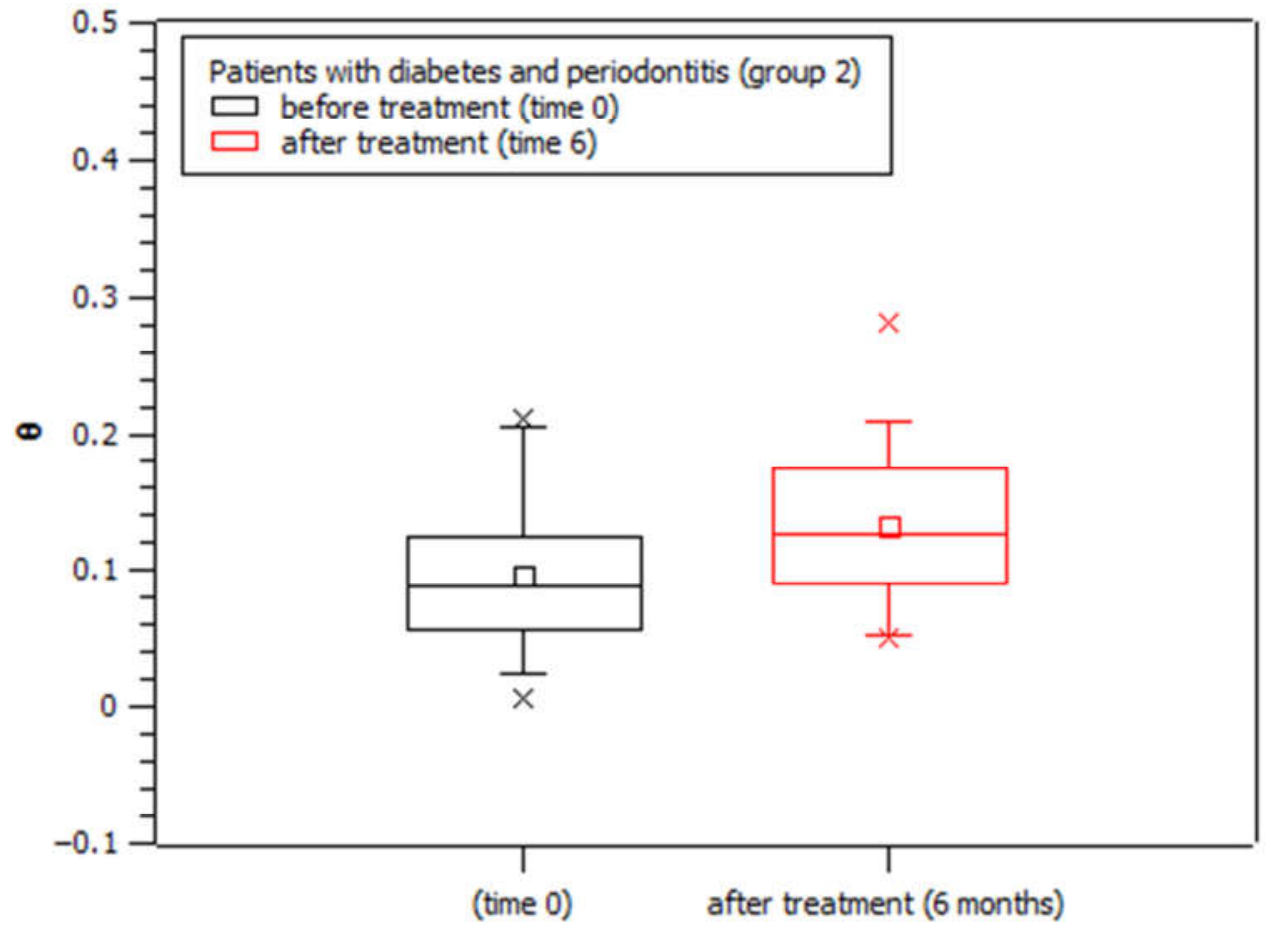

Figure 4-3: Box plot of the $\theta$ values of all patients from group 2 before and after 6 months of treatment $(p=0.029)$. 


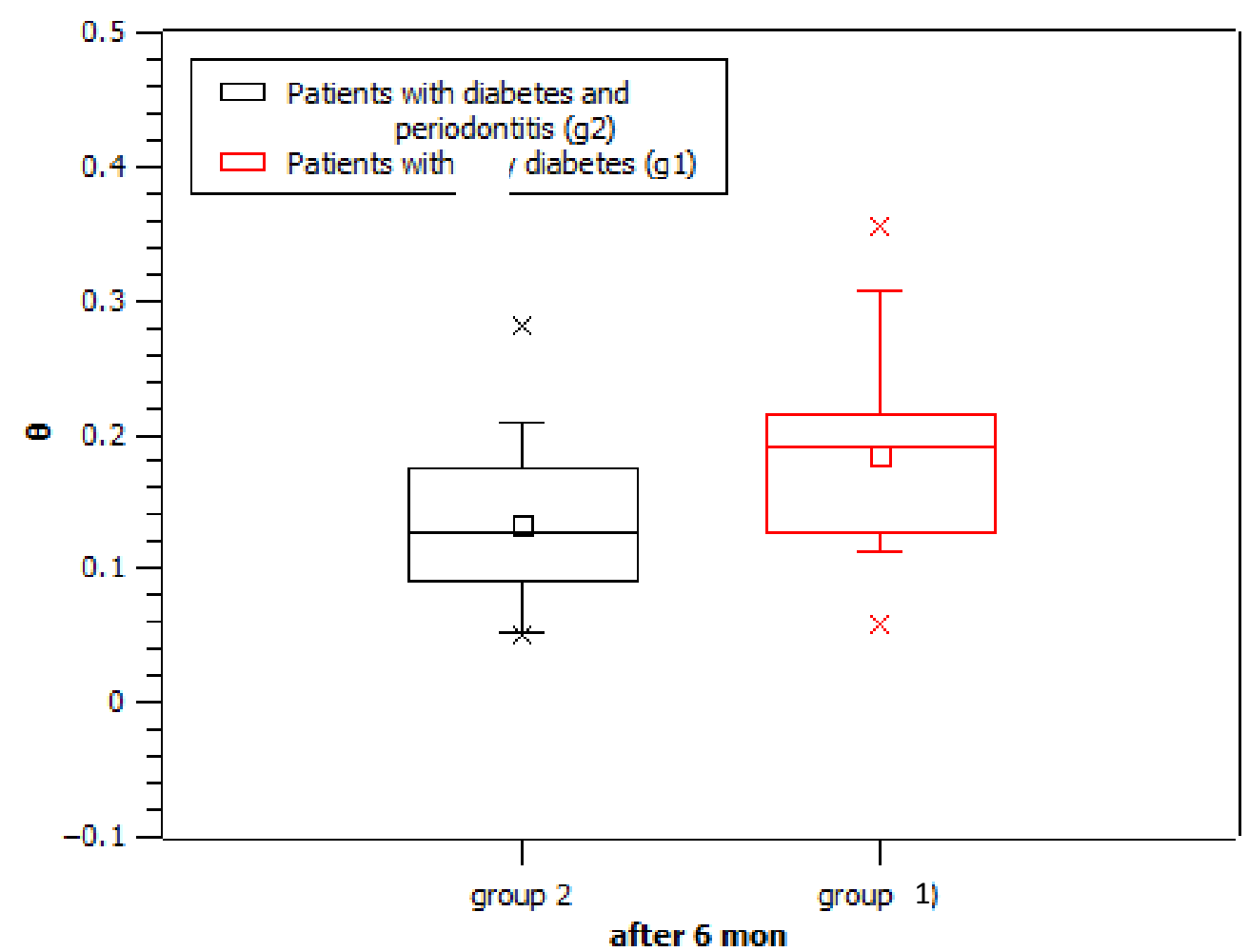

Figure 4-4: Box plot of the $\theta$ values of all the patient from group 1 and group 2 after 6 months (time 6$)(p=0.005)$.

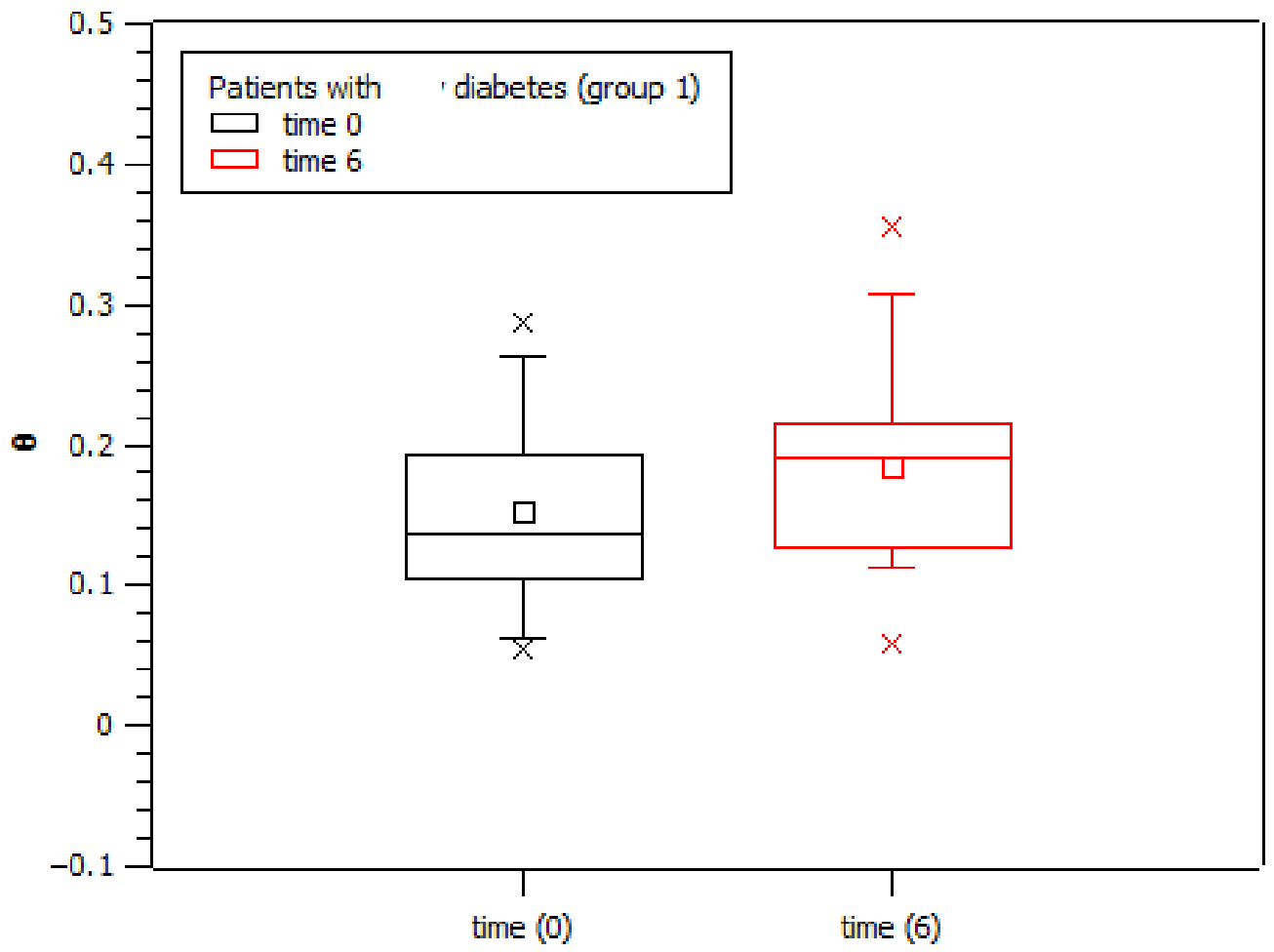

Figure 4-5: Box plot of the $\theta$ values of all patients from group 1 before and after 6 months $(p=0.084)$. 
The modification state of LDL particles is determined by the amplitude of the parameter $\theta$. The results showed that the modified LDL in the blood of patients with diabetes is lower than that of patients with diabetes and periodontitis. The results also showed that the degree of modification of LDL particles is diminished after the periodontal treatment, therefore it reduced the risk of cardiovascular disease.

In order to investigate the origin of the Z-scan results we will study the other parameters such as the liner absorption coefficient, the thermo-optic coefficient and the thermal conductivity, indicated in equation (2.7).

\section{4-2 Linear absorption}

Figure 4-6 shows some typical examples of optical absorption spectra of LDL samples from group 1 and group 2. The black points (extinction spectra) are obtained in the UV-Vis setup described in section 2-2-2, while the red line (scattering line) is a estimative for the Rayleigh scattering, obtained by fitting the equation (2.12) to the $\mathrm{UV}-\mathrm{V}$ is experimental points in the wavelength ranges where there is no absorption peaks. The absorption spectra (blue points) as a function of the wavelength are obtained subtracting the scattering curve from the UV-Vis extinction points. The results show the presence of three peaks in the absorption spectrum for both groups. The statistical analysis for all patients is done at the wavelength of $532 \mathrm{~nm}$, which is the wavelength of the laser used in the Z-Scan experiments. In order to discount the dependence of the absorption spectrum on the concentration we normalized the data in the same way we did for the Z-Scan amplitudes (equation (2.18)). 
To compare the contribution of the linear absorption in the Z-Scan amplitude, we calculate the absorption coefficient using equation (2.11), and then present their values in the box plot for all patients.
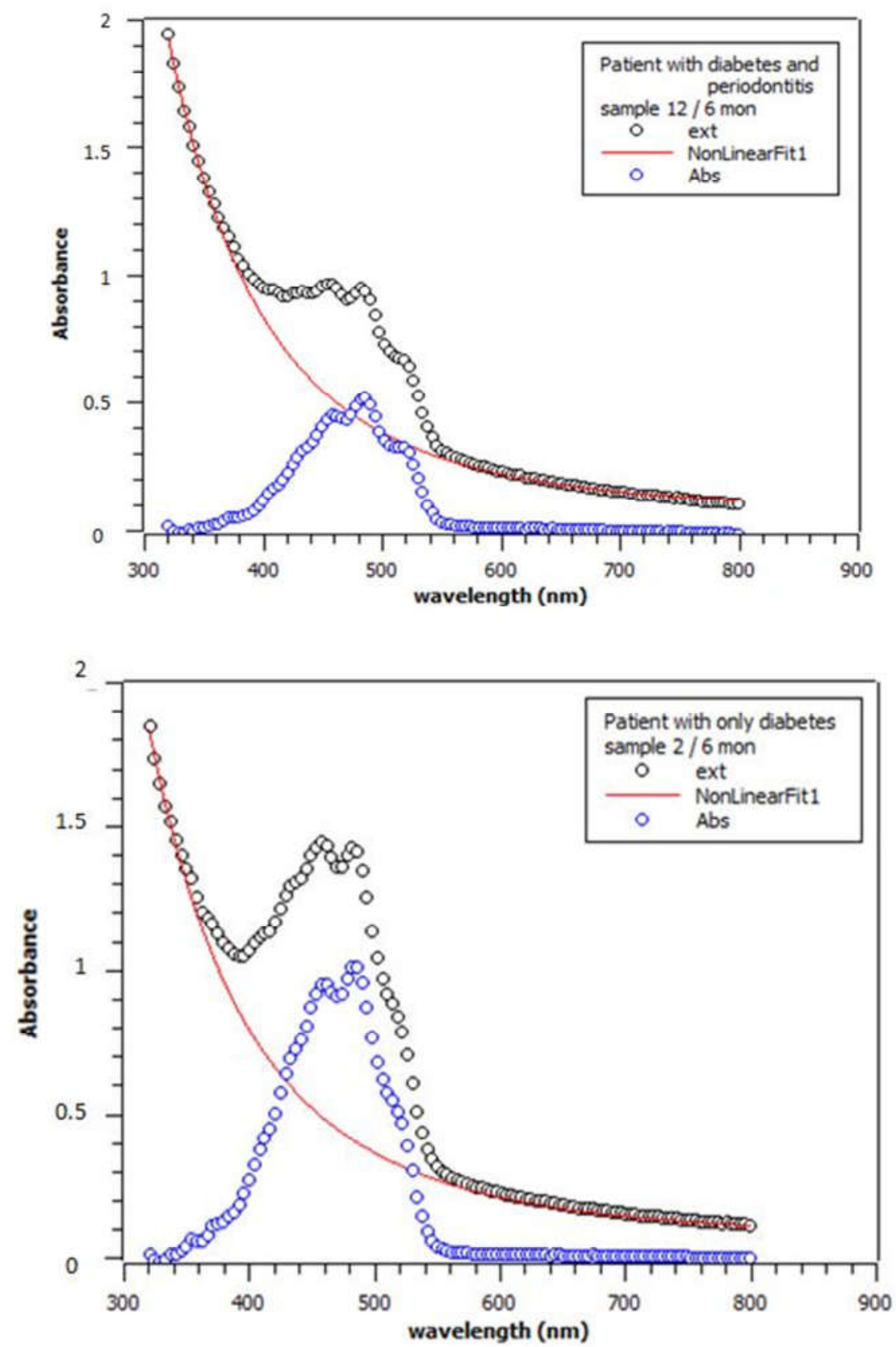

Figure 4-6: The absorbance (A) as a function of wavelength for LDL samples from some patients. 
In the beginning (time 0 ), the absorption coefficient values $(\alpha)$ of the 24 patients from group 1 and the 24 patients from group 2 are presented in the box plot shown in Figure 4-7. The results show that the mean value of $\alpha$ from the patients of group 1 is higher than group 2. This means that LDL particles of the patients with diabetes absorb light more than the patients with diabetes and periodontitis.

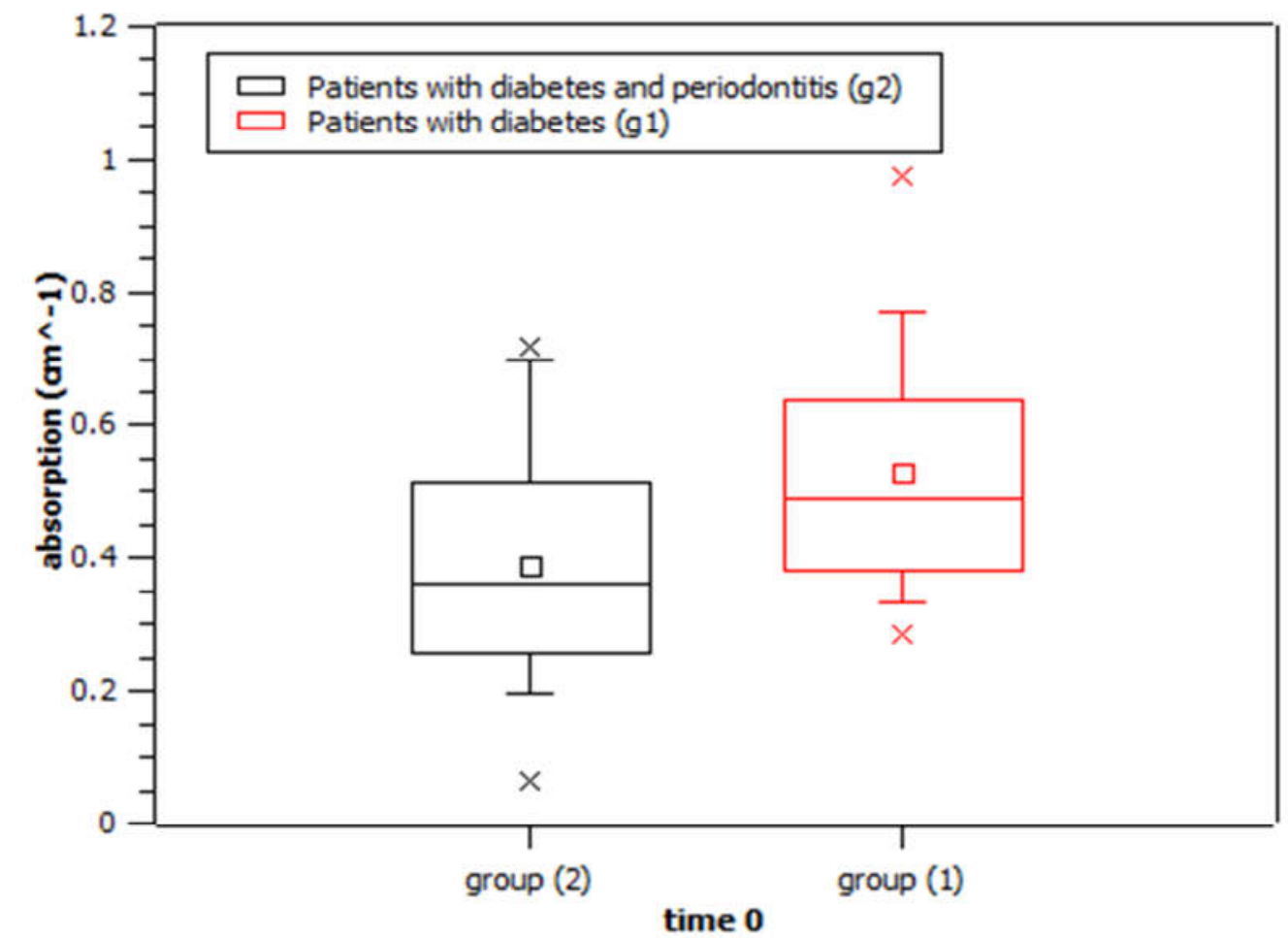

Figure 4-7: Box plot of the $\alpha$ values of all the patient from group 1 and group 2 in the beginning (time 0$)(p=0.007)$.

Figure 4-8 shows the box plot of the absorption coefficient values $(\alpha)$ of all the patients from group 2 before and after 6 months of the periodontal treatment. For group 2, the results show that there is no significant change in the mean value of $\alpha$ before and after 6 months of periodontal treatment. 


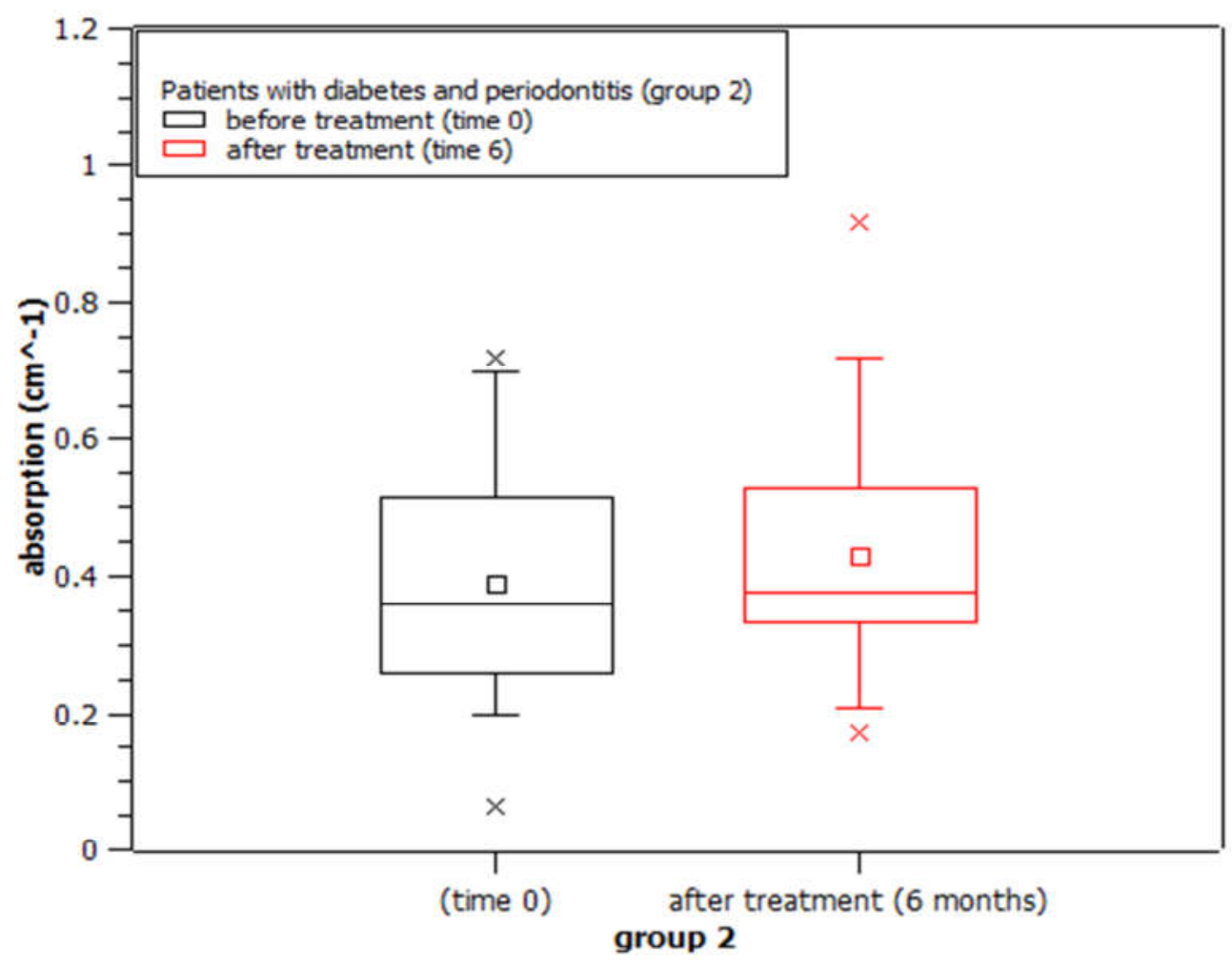

Figure 4-8: Box plot of the $\alpha$ values of all the patient from group 2 before and after 6 months of treatment $(p=0.388)$.

Figure 4-9 shows the box plot of the absorption coefficient values $\alpha$ of the two groups after 6 months. Group 1 didn't have any treatment while group 2 had a periodontal treatment after 6 months. The mean value of $\alpha$ is the same for both groups.

Figure 4-10 shows the box plot of the absorption coefficient values $\alpha$ of all the patients from group 1 in the beginning and after 6 months. For group 1, the results show that the mean value of $\alpha$ is the same before and after 6 months. 


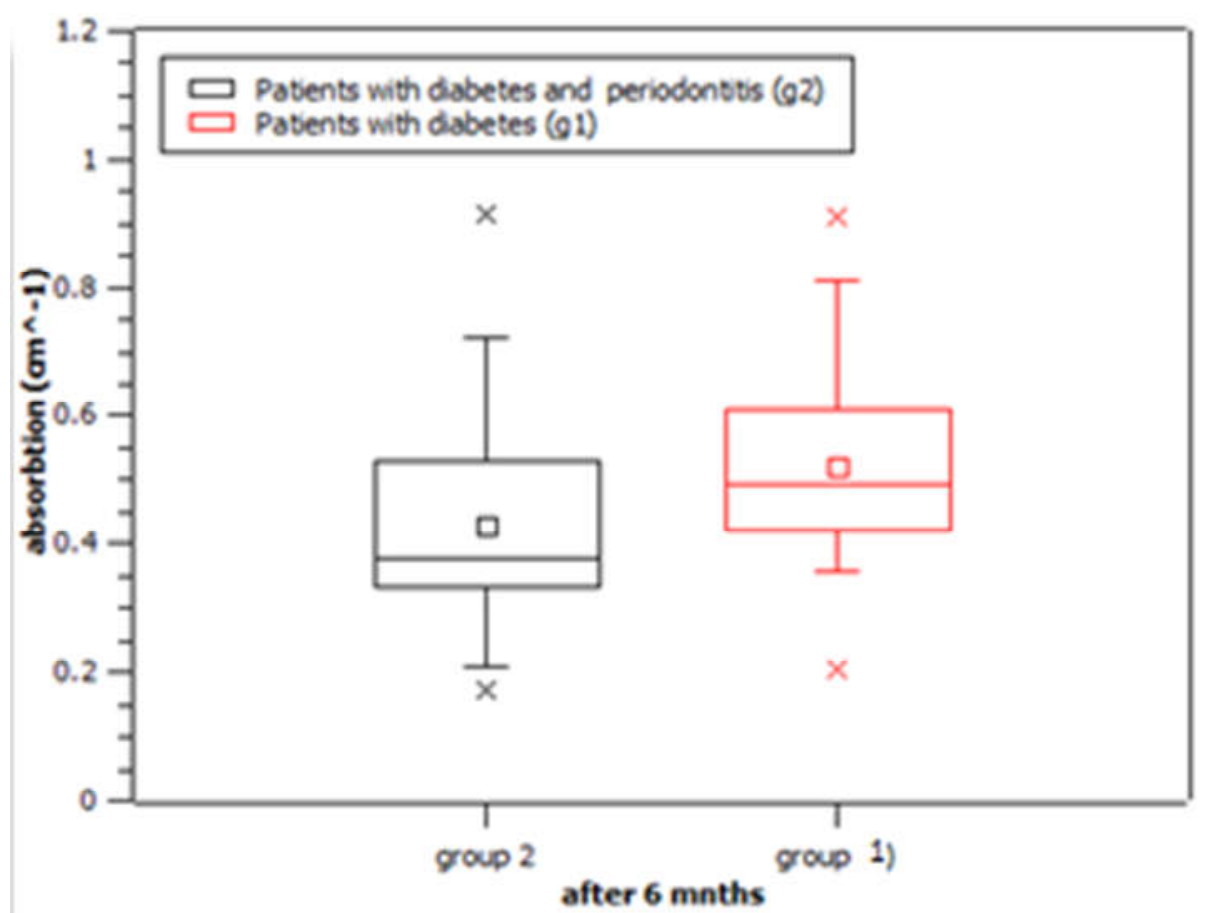

Figure 4-9: Box plot of the $\alpha$ values of all patients from group 1 and group 2 after 6 months $(p=0.068)$.

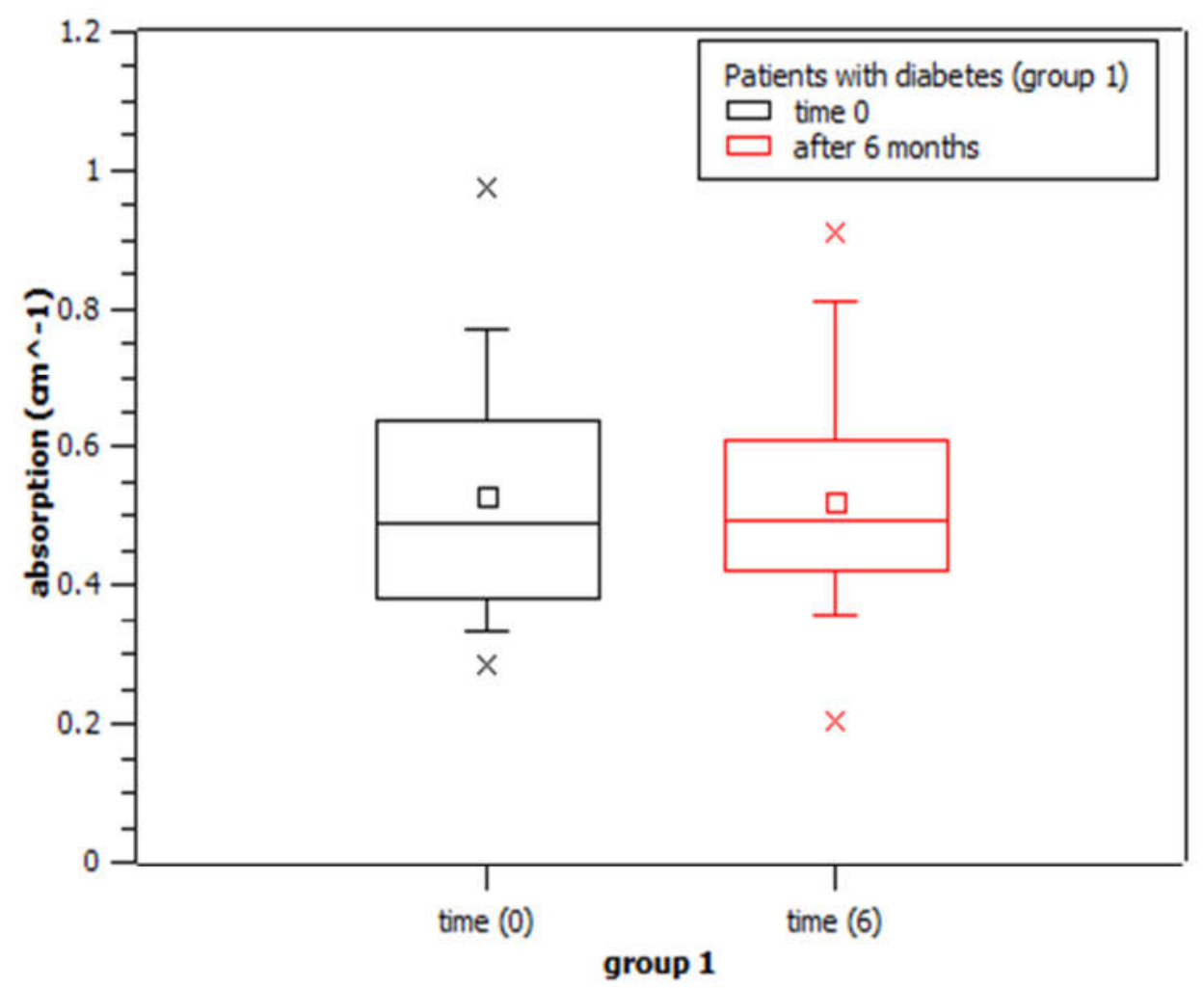

Figure 4-10: Box plot of the $\alpha$ values of all patients from group 1 before and after 6 months $(p=0.885)$. 


\section{4-3 Measurement of the thermo-optic coefficient}

Figure 4-11 shows the values obtained for the refractive index of LDL sample as a function of the temperature for one typical patient. The refractive index decreases with increasing the temperature for LDL particles solutions. The thermo-optic coefficient $(d n / d T)$ is obtained from the linear fit of the data. The mean value of $d n / d T$ for group 1 in time 0 is $(1.39 \pm 0.08) \times 10^{-4} / \mathrm{K}$. The mean value of $d n / d T$ for group 2 in time 0 is $(1.47 \pm 0.08) \times 10^{-4} / \mathrm{K}$. The mean value of $d n / d T$ for group 1 in time 6 is $(1.3 \pm 0.08) \times 10^{-4} / \mathrm{K}$. The mean value of $d n / d T$ for group 2 in time 6 is $(1.46 \pm 0.08) \times 10^{-4} / \mathrm{K}$. These results agree with those from Santos et al [71]. The thermo-optic coefficient is almost constant for LDL samples investigated.

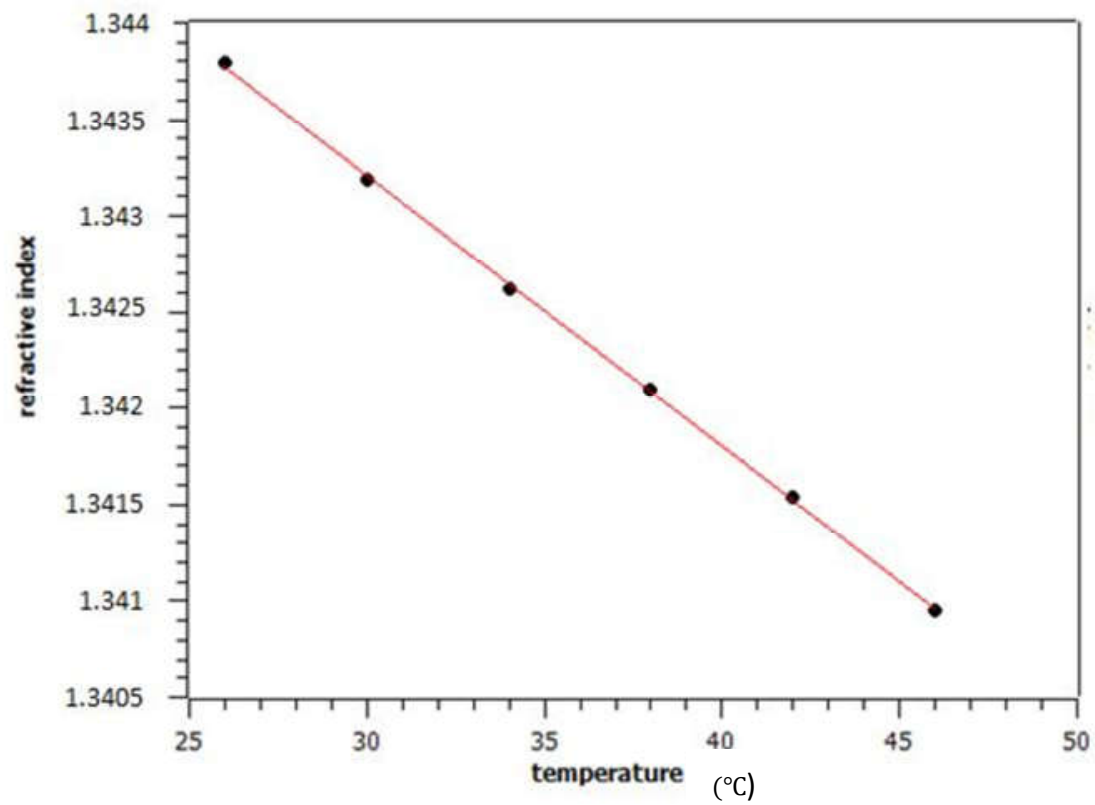

Figure 4-11: Temperature dependence of the refractive index of the LDL sample from one patient. 


\section{4-4 Thermal conductivity}

To verify the origin of the variation of the amplitude $(\theta)$ the thermal conductivity of LDL solutions is calculated. The other parameters $\left(\mathrm{P}, L_{e f f}\right)$ in the equation (2.7) are fixed. The results presented in section 4-3 show that there is no significant change in the thermo-optic coefficient values. If the linear absorption coefficient is the only physical parameter responsible for the changing in the amplitude of Z-scan, then thermal conductivity should be constant. The thermal conductivity is calculated according to equation (2.7).

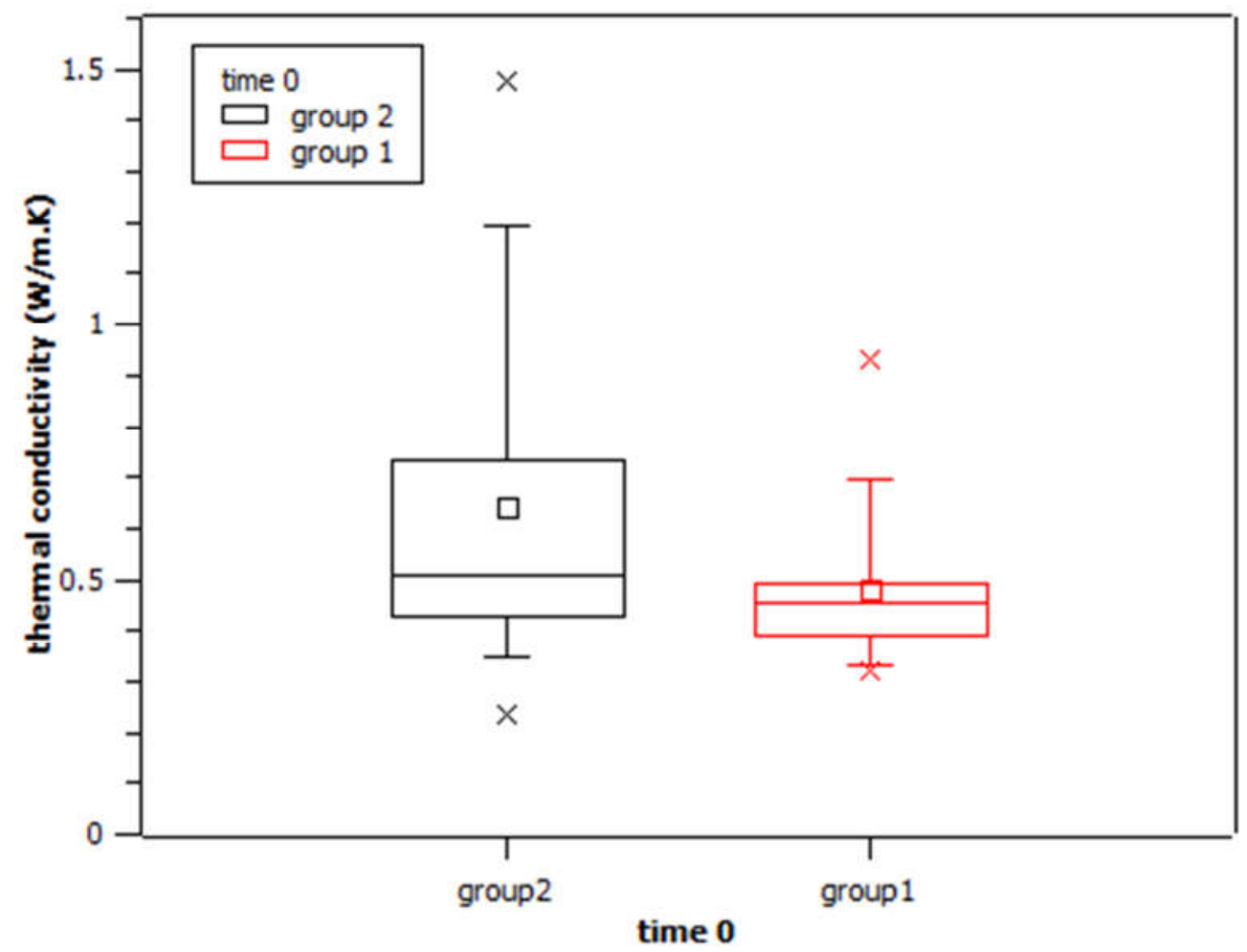

Figure 4-12: Box plot of the thermal conductivity $(k)$ values of all the patient from group 1 and group 2 in the beginning (time 0$)(p=0.008)$. 


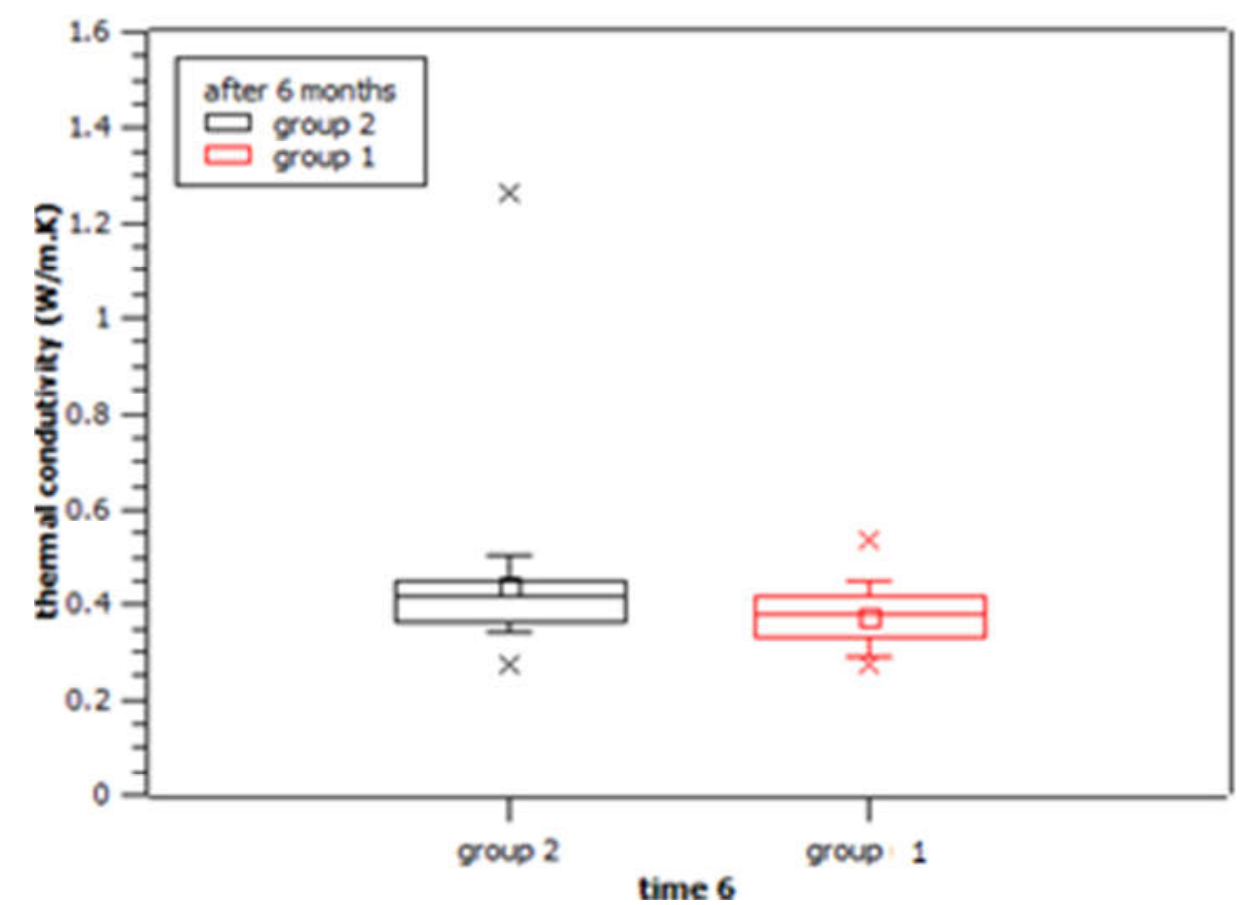

Figure 4-13: Box plot of the thermal conductivity $(k)$ values of all the patient from group 1 and group 2 after 6 months $(p<0.001)$.

The box plot of thermal conductivity values of all patients from group 1 and group 2 in time 0 and time 6 is shown in Figure 4-12 and 4-13 respectively. In time 0, the mean value of $\mathrm{k}$ from the patients of group 2 is higher than group 1 . After 6 months, the thermal conductivity values for group 2 is reduced, as shown in Figure 413. Although the mean value of $\mathrm{k}$ is still higher than group 1 but with a small difference.

For group 1, the box plot of thermal conductivity values in time 0 and after 6 months is shown in Figure 4-14. The results show that there is a change in the mean value between the beginning (time 0) and after 6 months (time 6), however, they are similar, taking into account the experimental errors.

For group 2, the box plot of thermal conductivity values in time 0 and after 6 months of treatment is shown in Figure 4-15. The results show that the mean value of $\mathrm{k}$ after 6 months of treatment is reduced. According to the results above the linear absorption 
coefficient and the thermal conductivity are the responsiple of changing the amplitude of Z-scan curves in LDL samples from patients with periodontitis and diabetes before and after the treatment. However, to improve our conclusions about the role of the thermal conductivity and the linear absorption coefficient in the changing of the Zscan amplitude, the experiments should be repeated after 12 months of the periodontal treatment.

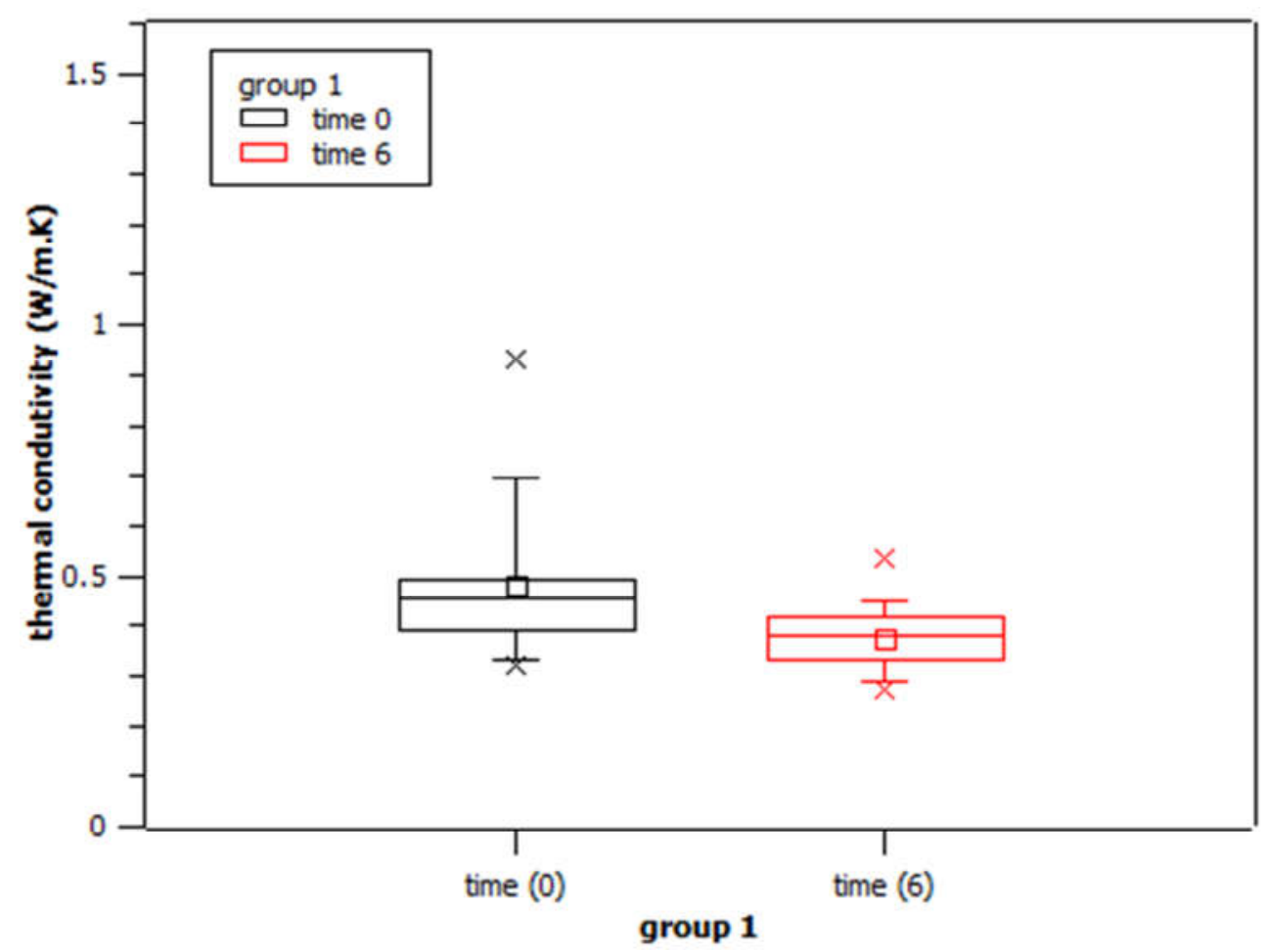

Figure 4-14: Box plot of the thermal conductivity $(k)$ values of all the patient from group 1 before and after 6 months $(p<0.001)$. 


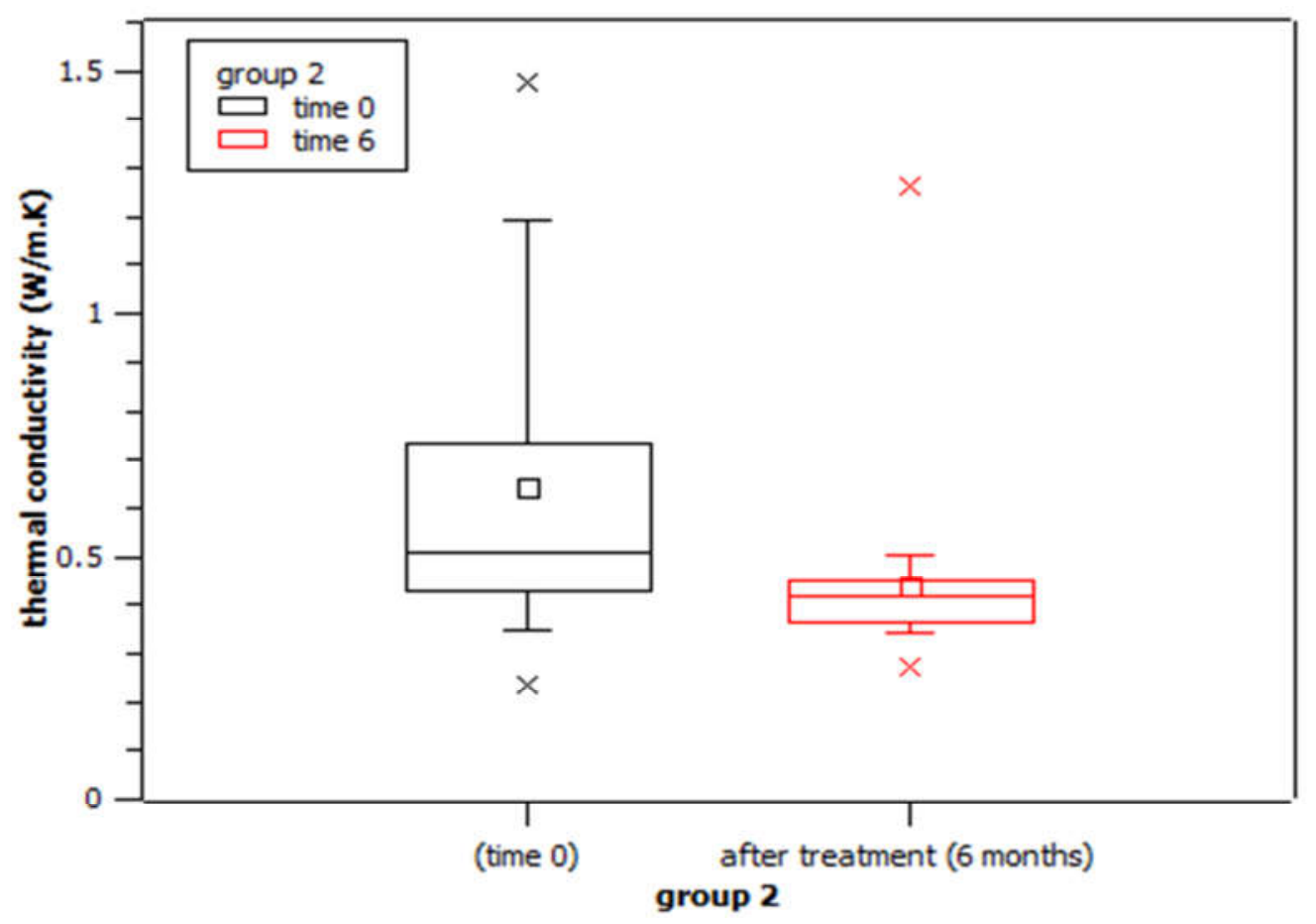

Figure 4-15: Box plot of the thermal conductivity $(k)$ values of all the patient from group 2 before and after 6 months of treatment $(p=0.008)$.

\section{4-5 Dynamic Light Scattering}

The dynamic light scattering experiment was employed to evaluate the LDL particle-size distributions of all the samples as well as the existence of particles' aggregates. For each sample, the measured autocorrelation curves were fitted using the NNLS method, resulting in particle-size distribution histograms weighted by number, volume, and intensity of scattered light, as shown in Figure 4-16 ( typical results for patient 48 at time 0 ). The average radius and distributions' standard deviation were calculated from the number-averaged particle-size distribution. The standard deviation was adopted as a measure of size polidispersity of the LDL sample. 
a)

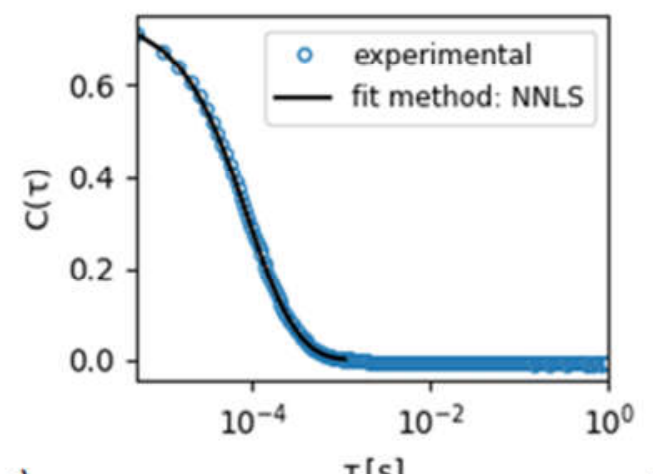

c)

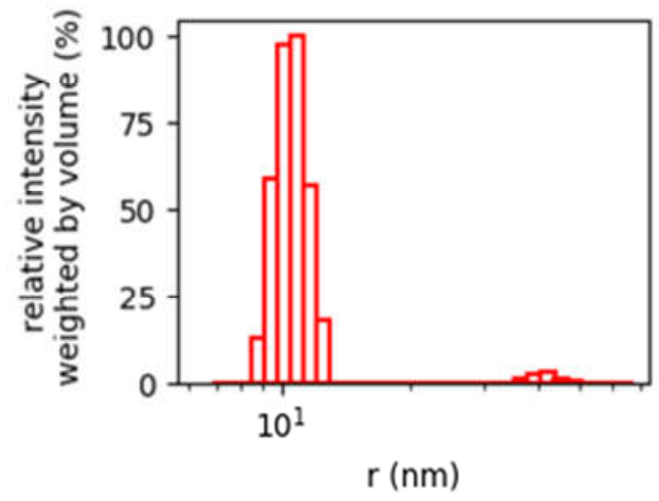

b)

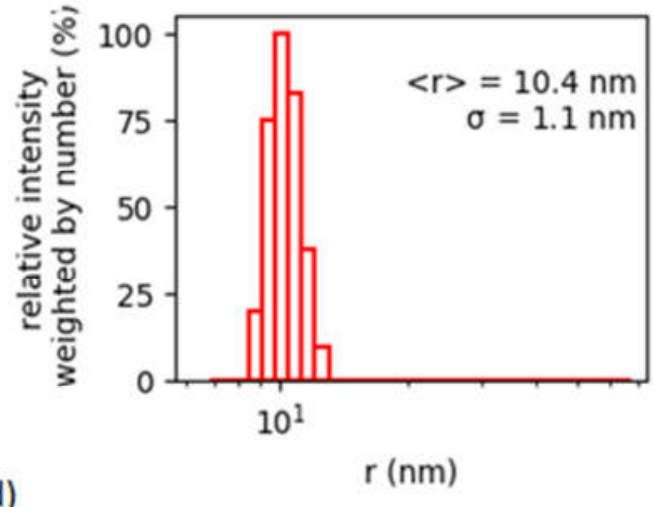

d)

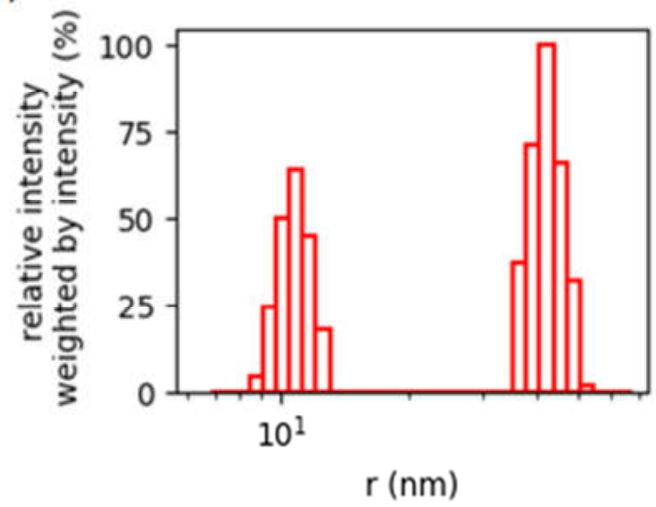

Figure 4-16: Measured (blue points) and fitted (black line) autocorrelation function using the NNLS method (a). Particle-size distribution histograms weighted by number (b), volume (c), and intensity of scattered light (d). Data and analysis done for patient 48 at time 0 .

Measurements were performed for samples from patients at time 0 and after 6 months. Figure 4-17(a) shows the box plot of the mean radius (weighted ) of LDL particles and Figure 4-17(b) shows the normalized intensity autocorrelation functions measured by DLS for the samples from groups 1 and 2 at time 0 . The results show that there is not a significant change in the sizes and polidispersities of LDL particles between the two groups. The mean value of the mean radius and standard deviation of the mean radius for group 1 is about $11.2 \pm 0.9 \mathrm{~nm}$ and for group 2 is about $11.6 \pm 1.0 \mathrm{~nm}$. The same results are obtained after 6 months where the mean value of the radius of LDL particles is the same for group 1 and group 2, as shown in Figure 4-18. The mean value of the mean radius and standard deviation for group 1 is about $10.4 \pm 0.7 \mathrm{~nm}$ and for group 2 is about $10.4 \pm 0.9 \mathrm{~nm}$. 
a)

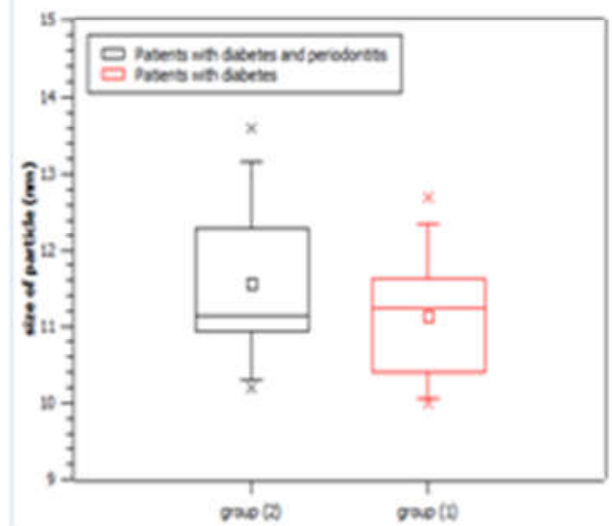

b)

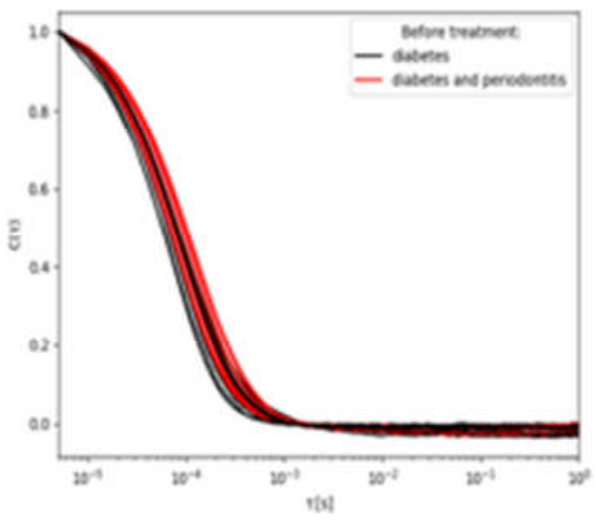

Figure 4-17: (a) Box plot of the sizes of LDL particles values of the patient from group 1 and group 2 in the beginning (time 0$)(p=0.355)$. (b) Intensity autocorrelation functions measured by dynamic light scattering (DLS) for the samples of patients.

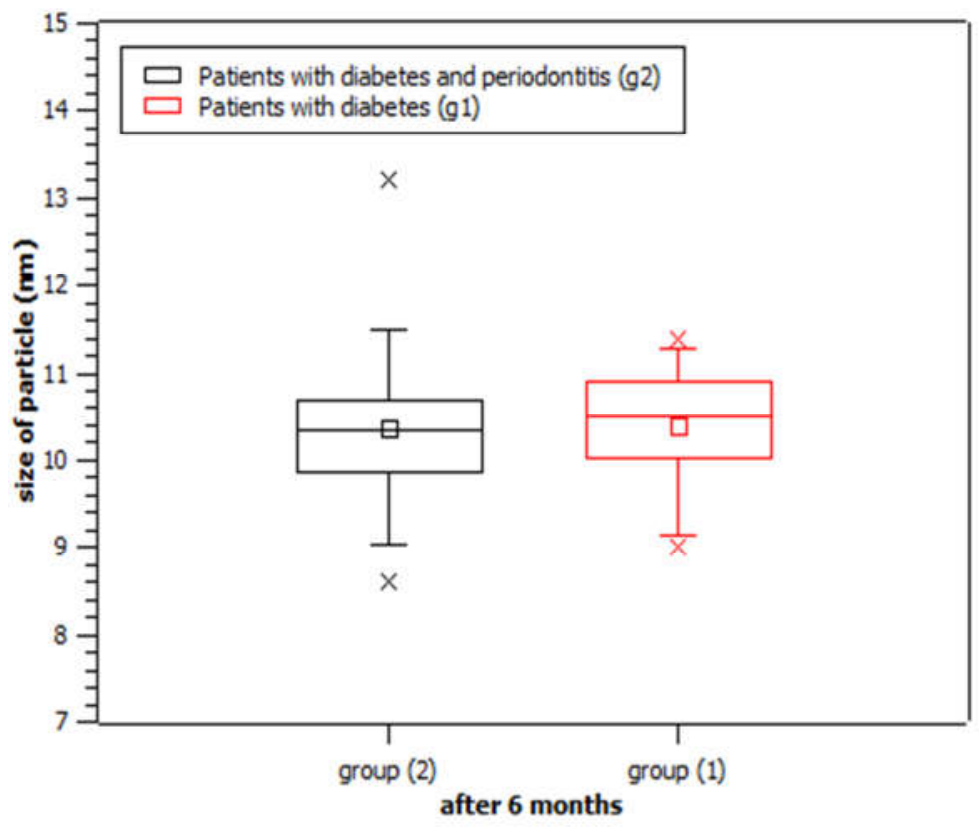

Figure 4-18: Box plot of the size of LDL particles values of the patient from group 1 and group 2 after 6 months $(p=0.846)$. 


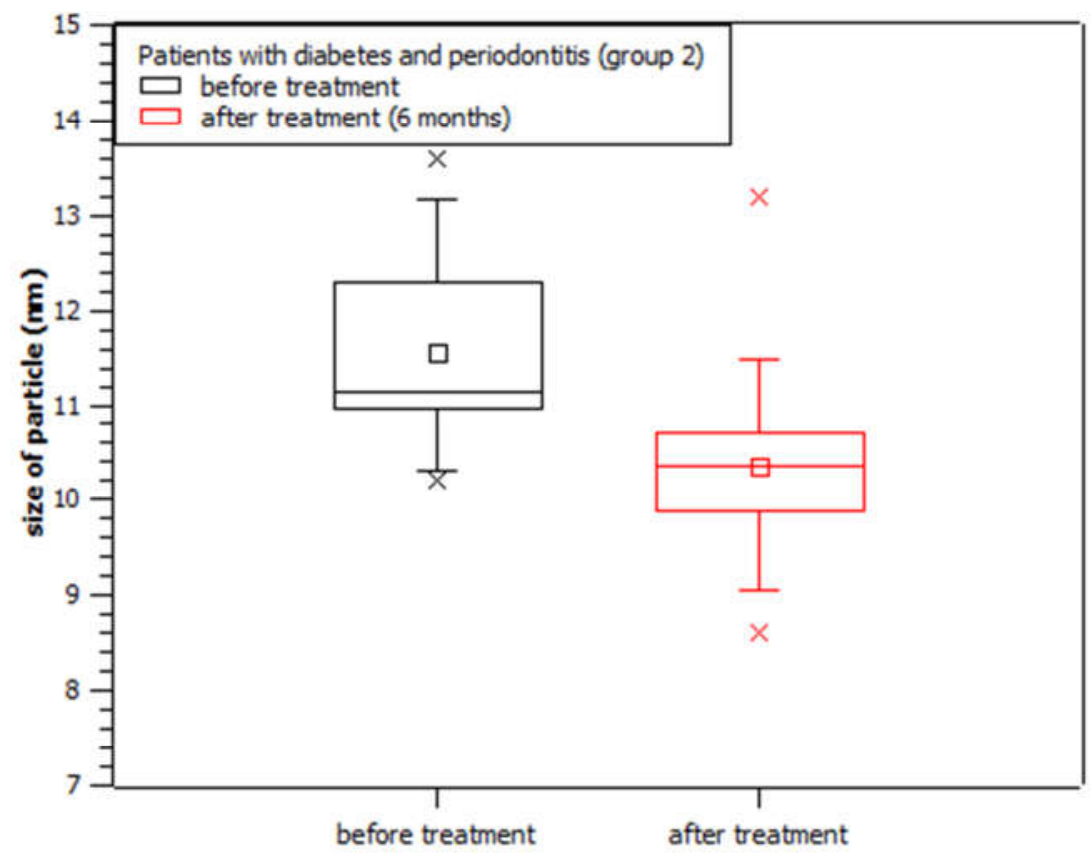

Figure 4-19: Box plot of the size of LDL particles values of the patient from group 2 before and after 6 months of treatment $(p=0.054)$.

Figure 4-19 shows the box plot of the mean radius (weighted) of LDL particle for group 2 before and after 6 months periodontal treatment. The results show that there is not a significant change in the mean value of the radius of LDL particles. Therefore, the sizes of LDL particles are about the same. The same results are obtained for group 1 and after 6 months as shown in Figure 4-20.

Also, observe that the size polidispersities (standard deviation of the distribution) presented in the number-weighed size distributions are small, though a very small fraction of very large aggregates exists in all samples, as shown in the intensities-weighted and volume-weighted sizes distributions.

As a result, the LDL nanoparticles size didn't change in patients with diabetes and patients with periodontitis and diabetes. 


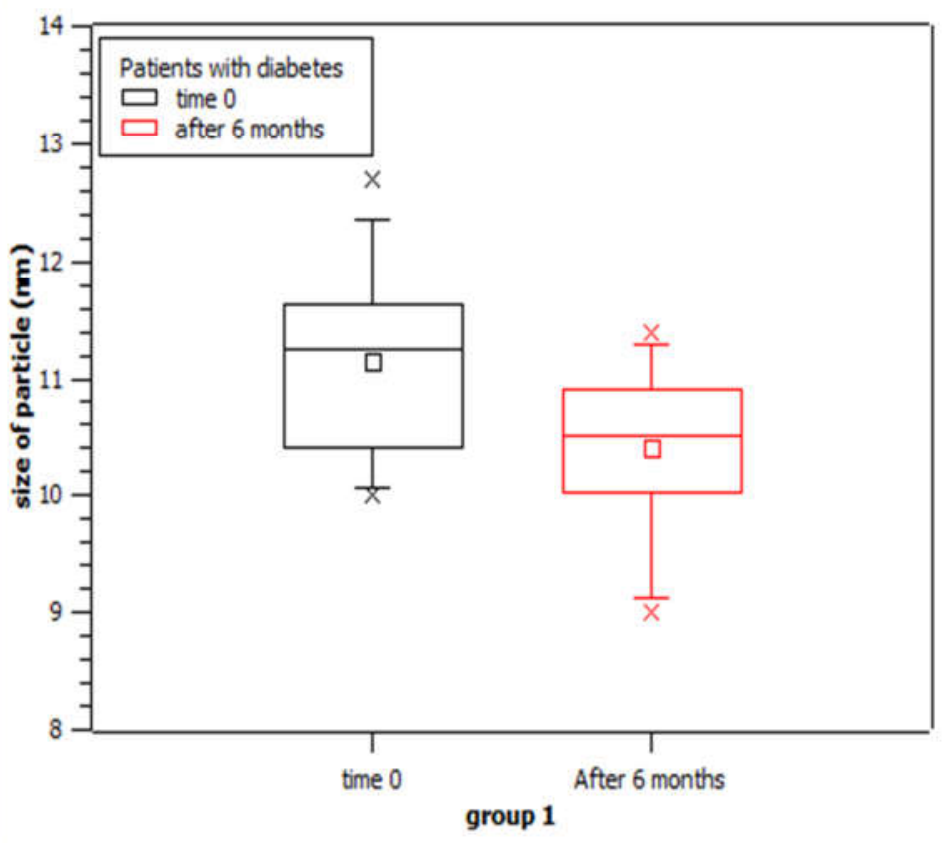

Figure 4-20: Box plot of the size of LDL particles values of the patient from group 1 before and after 6 months $(p=0.066)$. 


\section{Conclusion}

The Z-scan experiment is shown to be a good tool to differentiate the modification state of LDL particles between the diabetes patients with and without periodontitis. The experiment is also an easy way to evaluate the improvement of the quality of LDL particles after periodontal treatment. The results showed that the degree of modification of LDL from patients with periodontitis and diabetes is higher than in patients with diabetes and gingivitis. The linear absorption coefficient in patients with periodontitis and diabetes was lower than in patients with diabetes and gingivitis. The thermal conductivity seems to be higher in patients with periodontitis and diabetes than in patients with diabetes and gingivitis.

The degree of modification of LDL was shown to be reduced after the periodontal treatment in patients with periodontitis and diabetes. Therefore the risk of cardiovascular disease in diabetic patients has been reduced in the patients with periodontitis and diabetes by the treatment. The results showed that both groups of patients had a close value of the thermo-optical coefficient.

The size of LDL particles is about the same in both groups of patients. Also, this size didn't change after the periodontal treatment for group patients with periodontitis and diabetes. 


\section{References:}

[1] WHO, Library Cataloguing-in-Publication Data, Global status report on noncommunicable diseases, Geneva: World Health Organization, 2014;

[2] S. Eisenberg, Lipoproteins and Lipoprotein Metabolism, klin Wochenschr 61, 119-132 (1983);

[3] H. Esterbauer, J. Gebicki, H. Puhl, G. J"urgens, The role of lipid peroxidation and antioxidants in oxidative modication of LDL, Free Radic. Biol. Med. 13, 341-390 (1992);

[4] McNamara JR, Campos H, Ordovas JM, Peterson J, Wilson PWF, Schaefer EJ. Effect of gender, age and lipid status on low density lipoprotein subfraction distribution. Results from the Framingham Offspring Study. Arteriosclerosis 1987; 7: 483-490.

[5] Fisher W, Hammond MG, Mengel MC, Warmke GL. A genetic determinant of the phenotypic variance of the molecular weight of low density lipoprotein. Proc Natl Acad Sci USA 1975 ; 72: 2347-2351.

[6] L. Stryer, Bioqu' 1mica, 4a edi, , c ao, Guanabara Koogan (1996);

[7] Fisher WR. Heterogeneity of plasma low density lipoproteins manifestation of the physiologic phenomenon in man. Metabolism 1983; 32: 283-291.

[8] Chapman MJ, Laplaud PM, Luc G, et al. Further resolution of the low-density lipoprotein spectrum in normal human plasma: physicochemical characteristics of discrete subspecies separated by density gradient ultracentrifugation. J Lipid Res 1988; 29: 442-458.

[9] Baumstark MW, Kreutz W, Berg A, Frey I, Keul J. Structure of human LDL subfractions determined by X ray small angle scattering. Biochim Biophys Acta 1990; small, dense low density lipoprotein particles. J Clin Invest 1037: 48-57.

[10] Lorenzo Piemonte,About diabetes, The IDF Diabetes Atlas Eighth edition 2017, Published: 17 May 2018, https://idf.org/52-about-diabetes.html

[11] Nicholson G, Hall GM. Diabetes mellitus: new drugs for a new epidemic. Br J Anaesth 2011; 107: 65-73.

[12] WHO Library Cataloguing-in-Publication. Data Global report on diabetes. Geneva: World Health Organization; 2016.

[13] WHO. Definition, Diagnosis and Classification of Diabetes Mellitus and its Complications. Part 1: Diagnosis and Classification of Diabetes Mellitus (WHO/NCD/NCS/99.2). Geneva: World Health Organization; 1999.

[14] Emerging Risk Factors Collaboration. Sarwar N, Gao P, Seshasai SR, Gobin R, Kaptoge S, Di Angelantonio E. Diabetes mellitus, fasting blood glucose concentration, and risk of vascular disease: a collaborative meta-analysis of 102 prospective studies. Lancet. 2010 Jun 26;375(9733):2215-22. 
[15] Singh GM, Danaei G, Farzadfar F, Stevens GA, Woodward M, Wormser D et al. The age-specific quantitative effects of metabolic risk factors on cardiovascular diseases and diabetes: a pooled analysis. PLoS One 2013; 8(7):e65174.

[16] Brownlee M, Cerami A, Vlassara H. Advanced products of nonenzymatic glycosylation and the pathogenesis of diabetic vascular disease. Diabetes Metab Rev 1988;4(5):437-451.

[17] Benítez S, Pérez A, Sánchez-Quesada JL, et al. Electronegative low-density lipoprotein subfraction from type 2 diabetic subjects is proatherogenic and unrelated to glycemic control. Diabetes Metab Res Rev 2007;23(1):26-34.

[18] Dentino A, Lee S, Mailhot J, Hefti AF. Principles of periodontology. Periodontol 2000. $2013 \mathrm{Feb} ; 61(1): 16-53$.

[19] Bascones-Martınez A., Munoz-Corcuera M., Noronha S., Mota P., Bascones-Ilundain C., Campo-Trapero J. "Host defencemechanisms against bacterial aggression in periodontal disease: basic mechanisms," Medicina Oral, Patologia Oral y Cirugia Bucal, vol. 14, no. 12, pp. e680-e685, 2009.

[20] Sanz M, D'Aiuto F, Deanfield J, Fernandez-Avilés F. European workshop in periodontal health and cardiovascular disease-scientific evidence on the association between periodontal and cardiovascular diseases: A review of the literature. Eur Heart J Suppl 2010;12 Suppl B:B3-12.

[21] Kamil W., Al Habashneh R., Khader Y., Al Bayati L., Taani D. Effects of nonsurgical periodontal therapy on C-reactive protein and serum lipids in Jordanian adults with advanced periodontitis. Journal of Periodontal Research, vol. 46, no. 5, pp. 616-621, 2011.

[22] Casanova L, Hughes FJ, Preshaw PM. Diabetes and periodontal disease: A two-way relationship. Br Dent J 2014;217:433-7.

[23] Acharya AB, Thakur S, Muddapur MV. Effect of scaling and root planing on serum interleukin-10 levels and glycemic control in chronic periodontitis and type 2 diabetes mellitus. J Indian Soc Periodontol. 2015 Mar-Apr;19(2):188-93.

[24] Bascones-Martinez A, Matesanz-Perez P, Escribano-Bermejo M, González-Moles MÁ, Bascones-Ilundain J, Meurman JH. Periodontal disease and diabetes- Review of the Literature. Med Oral Patol Oral Cir Bucal. 2011 Sep 1;16(6):e722-9.

[25] Wu YY, Xiao E, Graves DT. Diabetes mellitus related bone metabolismo and periodontal disease. Int J Oral Sci 2015;26:63-72.

[26] Mealey BL, Ocampo GL. Diabetes mellitus and periodontal disease. Periodontol 2000 2007;44:127-53.

[27] Lee JH1, Oh JY, Youk TM, Jeong SN, Kim YT, Choi SH. Association between periodontal disease and non-communicable diseases: A 12-year longitudinal health-examinee cohort study in South Korea. Medicine (Baltimore). 2017 Jun;96(26):e7398.

[28]Holmstrup P, Damgaard C, Olsen I, Klinge B, Flyvbjerg A, Nielsen CH, Hansen PR. Comorbidity of periodontal disease: two sides of the same coin? An introduction for the 
clinician. J Oral Microbiol. 2017 Jun 14;9(1):1332710. doi: 10.1080/20002297.2017.1332710. eCollection 2017.

[29] Vadakkekuttical RJ, Kaushik PC, Mammen J, George JM. Does periodontal inflammation affect glycosylated haemoglobin level in otherwise systemically healthy individuals? - A hospital based study. Singapore Dent J. 2017 Dec;38:55-61.

[30] Monteiro AM, Jardini MA, Alves S, Giampaoli V, Aubin EC, Figueiredo Neto AM, Gidlund M. Cardiovascular disease parameters in periodontitis. J Periodontol. 2009 Mar;80(3):378-88.

[31] R. Stocker et al., "Role of oxidative modifications in atherosclerosis," Physiol. Rev. 84(4), 1381-1478 (2004).

[32] J. L. Witztum et al., "The oxidative modification hypothesis of atherosclerosis: does it hold for humans?," Trends. Cardiovasc. Med. 11(3-4), 93-102 (2001).

[33] S. Ehara et al., "Elevated levels of oxidized low density lipoprotein show a positive relationship with the severity of acute coronary syndromes," Circulation 103(15), $1955-1960$

(2001).

[34] P. Holvoet et al., "Circulating oxidized LDL is a useful marker for identifying patients with coronary artery disease," Arterioscler. Thromb. Vasc. Biol. 21(5), 844-848 (2001).

[35] M. Sheik-Bahae et al., "High-sensitivity, single-beam n2 measurements," Opt. Lett. 14(17), 955-957 (1989).

[36] M. Sheik-Bahae et al., "Sensitive measurement of optical nonlinearities usingasinglebeam,” IEEE J. Quantum Electron. 26(4),760-769(1990).

[37] S. L. Gómez et al., "Characterization of native and oxidized human low-density lipoproteins by the Z-scan technique," Chem. Phys. Lipids 132(2), 185-195 (2004).

[38] A.M. Monteiro, M.A.N. Jardini, V. Giampaoli, S. Alves, A.M. Figueiredo Neto, M. Gidlund, Measurement of the nonlinear optical response of low-density lipoprotein solutions from patients with periodontitis before and after periodontal treatment, evaluation of cardiovascular risk markers, J. Biomedical Optics 17, 115004(2012);

[39] P.B. de Melo, A.M. Nunes, L. Omena, S.M.S. do Nascimento, M.G.A. da Silva, M.R. Meneghetti, I.N. de Oliveira,Thermo-optical properties and nonlinear optical response of smectic liquid crystals containing gold nanoparticles,Phys. Rev. E92, 042504 (2015);

[40] V.M. Lenart, R.F. Turchiello, G.F. Goya, S.L. G'omez, Enhanced Thermal Lens Effect in Gold Nanoparticle-Doped Lyotropic Liquid Crystal by Nanoparticle Clustering Probed by Z-Scan Technique, Braz. J.Phys. 45, 213 (2015)

[41] R. Guti'errez Fuentes, J.F. S'anchez Ram' rrez, J.L. Jim'enez P'erez,J.A. Pescador Rojas, E. Ram'on-Gallegos, A. Cruz-Orea. Int. J.Thermophys. 28, 1048 (2007), 
[42] S.L. G'omez, V.M. Lenart, R.F. Turchiello, I.H. Bechtold, A.A.Vieira, H. Gallardo, Nonlinear optical properties of dye-doped E7 liquid crystals at the nematic-isotropic transition, Liq. Cryst. 43, 268 (2015);

[43] J.R.D. Pereira, A.M. Mansanares, A.J. Palangana, M.L. Baesso.Temperature dependence of the refractive index near the reentrant-isotropic-calamitic-nematic phase transition, Phys. Rev. E 64, 012701 (2001);

[44] A.A. Said, M. Sheik-Bahae, D.J. Hagan, T.W. Wei, J. Wang, J. Young, E.W. Van Stryland, Determination of bound-electronic and free-carrier nonlinearities in $\mathrm{ZnSe}$, GaAs, CdTe, and ZnTe, J. Opt. Soc. Am. B 9(3), 405-414 (1992);

[45] B. Yu, C. Zhu, F. Gan, Optical nonlinearity of Bi2O3 nanoparticles studied by Z scantechnique, J. Appl. Phys. 82(9), 4532-4537 (1997);

[46] L. Irimpan, B. Krishnan, V.P.N. Nampoori, P. Radhakrishnan, Linear and nonlinear optical characteristics of $\mathrm{ZnO}\{\mathrm{SiO} 2$ nanocomposites, Appl. Opt. 47(24), 4345-4351(2008);

[47] T. Kaplas, L. Karvonen, J. R onn, M.R. Saleem, S. Kujala, S. Honkanen, Y. Svirko,Nonlinear refraction in semitransparent pyrolytic carbon_lms, Opt. Mat. Express, 2(12), 1822-1827 (2012);

[48] Pramodini.S, THIRD-ORDER OPTICAL NONLINEARITY AND OPTICAL POWER LIMITING OF ORGANIC MATERIALS UNDER CW LASER ILLUMINATION, PhD Dissertation MANIPAL UNIVERSITY, MANIPAL- 576104 KARNATAKA, INDIA. July 2015.

[49] F. L. S. Cuppo, A. M. Figueiredo Neto, S. L. G'omez, and P. Palffy-Muhoray Thermallens model compared with the Sheik-Bahae formalism in interpreting Z-scan experiments on lyotropic liquid crystals, J. Opt. Soc. Am. B, 19, 1342-1348(2002);

[50] gomes cuppo Nonlinear Optical Properties of Liquid Crystals Probed by Z-scan Technique.

[51] J. P. Gordon, R. C. C.Leite, R. S.Moore, S. P. S. Porto, and J. R. whinnery, Longtransient effects in lasers with inserted liquid samples, J. Appl. Phys. 36, 3 (1965);

[52] C. Hu and J. R. Whinnery, New Thermooptical Measurement Method and a Comparison with Other Methods, Appl. Opt. 12, 72 (1973).

[53] J. R. Whinnery, Laser measurement of optical absorption in liquids, Acc. Chem. Res. 7, 225

[54] F. L. S. Cuppo, and A. M. Figueiredo Neto, Thermal and Nonlinear Optical Properties of Potassium Laurate/Water Solutions at Amphiphilic Concentrations around the Critical 
Micellar Concentration, A Laser Gaussian Single Beam Experiment in Millisecond Timescales, Soc. Chm. Am, 18, 9647-9653 (2002);

[55] S. Alves, A. Bourdon, and A. M. Figueiredo Neto, Generalization of the thermal lens model formalism to account for thermodiffusion in a single-beam Z-scan experiment: determination of the Soret coefficient, J. Opt. Soc. Am. B, 20, 713-718 (2003);

[56] S. J. Sheldon, L. V. Knight, and J. M. Thorne, Laser-induced thermal lens effect: a new theoretical model, Appl. Opt. 21, 1663(1982);

[57] J. Shen, R. D. Lowe, and R. D. Snook, A model for cw laser induced mode-mismatched dual-beam thermal lens spectrometry, Chem. Phys, 165, 385-396 (1992);

[58] C. A. Carter and J. M. Harris, Comparison of models describing the thermal lens effect, Appl. Opt, 23, 476-481 (1984);

[59] A. L. Sehnem, D. Espinosa, E. S. Goncalves, and A. M. Figueiredo Neto, Thermal Lens Phenomenon Studied by the Z-Scan, Technique, Measurement of the Thermal Conductivity of Highly Absorbing Colloidal Solutions, J. Braz. Phys, 46 , 547-555 (2016);

[60] D. H. G. Espinosa, Efeito de lente térmica e não- linearidades ópticas do silício amorfo hidrogenado dopado com fósforo, Dissertation, USP (2011)

[61] M. Derakhshesh, M. R. Gray, and G. P. Dechaine, Dispersion of Asphaltene Nanoaggregates and the Role of Rayleigh Scattering in the Absorption of Visible Electromagnetic Radiation by These Nanoaggregates, Soc. Chm. Am, 27, 680-693 (2013);

[62] W. Schärtl, Light Scattering from Polymer Solutions and Nanoparticle Dispersions, Springer Laboratory, Springer-Verlag Berlin Heidelberg,57-8(2007);

[63] R. Xu, Particle Characterization, Light Scattering Methods, Springer Science, (2001);

[64] C. L. P. Oliveira Santos ET AL, Effect of Oxidation on the Structure of Human Lowand High-Density Lipoproteins, Biophys J, 106, 2595-2605, (2014);

[65] M. D. Spirito ET AL , Low Density Lipoprotein Aged in Plasma Forms Clusters Resembling

Subendothelial Droplets: Aggregation via Surface Sites, Biophys J, 90, 4239-4247 (2006);

[66] S. Trirongjitmoash ET AL, Fraction estimation of small, dense LDL using autocorrelation function of dynamic light scattering, Opt Express, 18 (2010)

[67] Tonetti MS, Claffey N. Advances in the progression of periodontitis and proposal of definitions of a periodontitis case and disease progression for use in risk factor research. 
Group C consensus report of the 5th European Workshop in Periodontology. J Clin Periodontol. 2005;32 Suppl 6:210-3.

[68] Havel RJ, Eder HA, Bragdon JH. The distribution and chemical composition of ultracentrifugally separated lipoprotein in human serum. J Clin Invest. 1955;32:1345-53.

[69] Smith PK, Krohn RI, Hermanson GT, Mallia AK, Gartner FH, Provenzano MD, Fujimoto EK, Goeke NM, Olson BJ, Klenk DC. Measurement of protein using Bicinchoninic Acid. Anal. Biochem. 1985 Oct;150(1):76-86.

[70] SPSS INCORPORATION. Statistical Package for the Social Sciences for Windows Student Version/ SPSS [computer program]. Release 16.0. Chicago: Marketing Department; 2007.

[71] P. R. Santos,T. C. Genaro-Mattos, A. M. Monteiro,S. Miyamoto, and A. M. Figueiredo Neto, Behavior of the thermal diffusivity of native and oxidized human low-density lipoprotein solutions studied by the Z-scan technique, J Biomed Opt, 17, 105003 (2012); 